\title{
The effects of Pilates vs. aerobic training on cardiorespiratory fitness, isokinetic muscular strength, body composition, and functional tasks outcomes for individuals who are overweight/obese: A clinical trial
} \author{
Vancini $^{4}$, Naryana Mascarin ${ }^{1}$, Marilia S Andrade ${ }^{\text {Corresp. } 1}$ \\ 1 Departamento de Fisiologia, Universidade Federal de São Paulo, São Paulo, São Paulo, Brazil \\ 2 Faculdade de Educação Física e Dança, Universidade Federal de Goiás, Goiânia, Goiás, Brazil \\ 3 Escola de Artes, Ciências e Humanidades, Universidade de São Paulo, São Paulo, São Paulo, Brazil \\ 4 Centro de Educação Física e Desportos, Universidade Federal do Espírito Santo, Vitória, Espírito Santo, Brazil \\ Corresponding Authors: Claudio Andre B de Lira, Marilia S Andrade \\ Email address: andre.claudio@gmail.com, marilia1707@gmail.com
}

Angeles Rayes ${ }^{1}$, Claudio Andre B de Lira ${ }^{\text {Corresp., }}{ }^{2}$, Ricardo B Viana ${ }^{2}$, Ana A. Benedito-Silva ${ }^{3}$, Rodrigo L

Background: Some studies have been conducted to verify the effects of Pilates for individuals who are obese, but conclusive results are not yet available due to methodological concerns. The present study aims to verify and compare the effects of Pilates and aerobic training on cardiorespiratory fitness, isokinetic muscular strength, body composition, and functional task outcomes for individuals who are overweight/obese.

Methods: Of the sixty participants, seventeen were allocated to the control group, since the intervention protocol (Pilates or walking sessions) was during their working hours. The remaining 44 participants were randomly allocated to one of two experimental groups (Pilates $(n=22)$ or aerobic groups $(n=21)$. The Pilates and aerobic groups attended $60-$ min exercise sessions, three times per week for 8 weeks. The aerobic group performed walking training at a heart rate corresponding to the ventilatory threshold. The Pilates group performed exercises on the floor, resistance apparatus, and 1-kg dumbbells. The control group received no intervention. All volunteers were evaluated at the beginning and end of the intervention. The following assessments were conducted: food intake, cardiorespiratory maximal treadmill test, isokinetic strength testing, body composition and anthropometry, abdominal endurance test, trunk extensor endurance test, flexibility test and functional (stair and chair) tests. Results: There was no significant difference pre- and post-intervention in calorie intake $[F(2,57)=0.02744, p=0.97)]$. A significant improvement in oxygen uptake at ventilatory threshold $(p=0.001 ; d=0.60)$, respiratory compensation point $(p=0.01 ; d=0.48)$, and maximum effort $(p=0.01 ; d=0.33$ ) was observed only in the Pilates group. Isokinetic peak torque for knee flexor and extensor muscles did not change for any groups. Lean mass ( $p=0.0005 ; d=0.19)$ and fat mass 
( $p=0.0001 ; d=0.19)$ improved only in the Pilates group. Waist and hip circumference measurements decreased similarly in both experimental groups. Abdominal test performance improved more in the Pilates group $(p=0.0001 ; d=1.69)$ than in the aerobic group ( $p=0.003 ; d=0.95)$. Trunk extensor endurance and flexibility improved only in the Pilates group ( $p=0.0003 ; d=0.80$ and $p=0.0001 ; d=0.41$, respectively). The Pilates group showed greater improvement on the chair and stair tests $(p=0.0001 ; d=1.48$ and $p=0.003$; $d=0.78$, respectively) than the aerobic group ( $p=0.005 ; d=0.75$ and $p=0.05 ; d=0.41$, respectively). Conclusion: Pilates can be used as an alternative physical training method for individuals who are overweight or obese since it promotes significant effects in cardiorespiratory fitness, body composition, and performance on functional tests. 
1 Research Article

2

3 The effects of Pilates vs. aerobic training on cardiorespiratory fitness, isokinetic muscular

4 strength, body composition, and functional tasks outcomes for individuals who are

5 overweight/obese: A clinical trial

6

7 Running head: Pilates for overweight and obesity

8

9 Angeles Rayes ${ }^{1}$, Claudio Andre B. de Lira ${ }^{2}$, Ricardo B. Viana ${ }^{2}$, Ana A. Benedito-Silva ${ }^{3}$, Rodrigo

L. Vancini ${ }^{4}$, Naryana Mascarin 1 , Marilia S. Andrade ${ }^{1}$

11

12 'Departmento de Fisiologia, Universidade Federal de São Paulo (UNIFESP), São Paulo (SP), 13 Brazil.

$14{ }^{2}$ Faculdade de Educação Física e Dança, Universidade Federal de Goiás (UFG), Goiânia (GO), 15 Brazil.

$16{ }^{3}$ Escola de Artes, Ciências e Humanidades, Universidade de São Paulo (USP), São Paulo (SP), 17 Brazil

$18{ }^{4}$ Centro de Educação Física e Desportos, Universidade Federal do Espírito Santo (UFES), Vitória 19 (ES), Brazil.

21 Corresponding author:

22 Claudio Andre Barbosa de Lira

23 E-mail: andre.claudio@gmail.com 
25

\section{ABSTRACT}

Background: Some studies have been conducted to verify the effects of Pilates for individuals who are obese, but conclusive results are not yet available due to methodological concerns. The present study aims to verify and compare the effects of Pilates and aerobic training on cardiorespiratory fitness, isokinetic muscular strength, body composition, and functional task outcomes for individuals who are overweight/obese.

Methods: Of the sixty participants, seventeen were allocated to the control group, since the intervention protocol (Pilates or walking sessions) was during their working hours. The remaining 44 participants were randomly allocated to one of two experimental groups (Pilates $(\mathrm{n}=22)$ or aerobic groups $(\mathrm{n}=21)$. The Pilates and aerobic groups attended 60 -min exercise sessions, three times per week for 8 weeks. The aerobic group performed walking training at a heart rate corresponding to the ventilatory threshold. The Pilates group performed exercises on the floor, resistance apparatus, and 1-kg dumbbells. The control group received no intervention. All volunteers were evaluated at the beginning and end of the intervention. The following assessments were conducted: food intake, cardiorespiratory maximal treadmill test, isokinetic strength testing, body composition and anthropometry, abdominal endurance test, trunk extensor endurance test, flexibility test and functional (stair and chair) tests. Results: There was no significant difference pre- and post-intervention in calorie intake $[\mathrm{F}(2,57)=0.02744, \mathrm{p}=0.97)]$. A significant improvement in oxygen uptake at ventilatory threshold $(\mathrm{p}=0.001 ; \mathrm{d}=0.60)$, respiratory compensation point $(p=0.01 ; d=0.48)$, and maximum effort $(p=0.01 ; d=0.33)$ was observed only in the Pilates group. Isokinetic peak torque for knee flexor and extensor muscles did not change for any groups. Lean mass $(\mathrm{p}=0.0005 ; d=0.19)$ and fat mass $(\mathrm{p}=0.0001 ; d=0.19)$ improved only in the Pilates group. Waist and hip circumference measurements decreased similarly in both experimental groups. Abdominal test performance improved more in the Pilates group $(\mathrm{p}=0.0001 ; d=1.69)$ than in the aerobic group $(\mathrm{p}=0.003 ; d=0.95)$. Trunk extensor endurance and flexibility improved only in the Pilates group $(\mathrm{p}=0.0003 ; d=0.80$ and $\mathrm{p}=0.0001$; $\mathrm{d}=0.41$, respectively). The Pilates group showed greater improvement on the chair and stair tests $(\mathrm{p}=0.0001 ; d=1.48$ and $\mathrm{p}=0.003 ; d=0.78$, respectively) than the aerobic group $(\mathrm{p}=0.005 ;$ $d=0.75$ and $\mathrm{p}=0.05 ; d=0.41$, respectively). 
54 Conclusion: Pilates can be used as an alternative physical training method for individuals who

55 are overweight or obese since it promotes significant effects in cardiorespiratory fitness, body composition, and performance on functional tests.

57

58

59

60

61

62

63

64

65

66

67

68

69

\section{INTRODUCTION}

Obesity constitutes an important threat to global public health in terms of prevalence, incidence, and economic burden (Tremmel et al., 2017). According to the World Health Organization (WHO), more than 1.9 billion adults aged 18 years and older are overweight (WHO, 2015); of these, over 650 million are obese, and this number is growing exponentially worldwide (WHO, 2015). The most common cause of obesity identified in a recent review study was “combined physical inactivity and inadequate diet” (Ross, Flynn \& Pate, 2016). In spite of obesity being associated with an increased risk of premature death (Xiao \& Fu, 2015), in a recent review, Barry et al. (2014) concluded that unfit individuals with lower cardiorespiratory levels had twice the risk of mortality than fit individuals, regardless of body mass index (BMI). Although fatness is associated with several chronic diseases, data suggest that the low cardiorespiratory levels in obese people are worse for mortality risk than the fatness, (Barry et al., 2014), so physical activity programs for obese people should aim to improve physical fitness in addition to reducing total body mass.

There is no consensus in the literature as to whether physical activity is important for total body mass loss and improved body composition (Pedersen \& Saltin, 2015). The amount of body mass loss associated with the introduction of physical activity probably is not very high, considering the much lower degree of energy imbalance created by increased physical activity than by food restriction (Wing, 1999). However, several studies showed that physical training promotes a decrease in fat mass and in abdominal fatness, unlike diet intervention, which, in a harmful sideeffect of very restrictive calorie diets, also promotes a fat-free mass loss (Ross \& Janssen, 1999).

Moderate walking or jogging programs have traditionally been suggested to improve variables related to health (Ross et al., 2000; Pedersen \& Saltin, 2015; ), however, in a recent review, Mabire et al. (2017) demonstrated that the effects of the walking training depend on the sex, age and body mass index. For obese people it is also difficult to exercise with weight bearing activities without supervision, because obesity is an important risk factor involved in knee pain and initiation and progression of osteoarthritis (Santangelo et al., 2016). Adherence levels to traditional aerobic programs are not very high, particularly if they are monotone and potentially 
painful for obese individuals (Burgess et al., 2017).

Alternative exercises should therefore be considered, such as planning enjoyable activities or amusing, non-weight-bearing exercises that consider the importance of improving aerobic fitness, muscular condition, and body composition. One option is Pilates, which is a body/mind training regime involving a variety of exercises for core stability, muscular strength, flexibility, attention to muscle control, posture, and breathing (Penelope, 2002; Aladro-Gonzalvo et al., 2012; Küçükçakır, Altan \& Korkmaz, 2013; de Oliveira Francisco, de Almeida Fagundes \& Gorges, 2015). Because of this variety, Pilates avoids monotony can be performed in a seated, standing, or lying position, thus protecting the knee joints and avoiding weight bearing. Pilates apparatus, such as the Reformer, are constructed in a manner that can accommodate many human anatomic variations and can be adjusted such that similar properties of movement sequencing can be applied to a variety of body types and limb/torso lengths (Muscolino \& Cipriani, 2004), which is of fundamental importance for obese people.

Some studies have been conducted to verify the effects of Pilates for obese individuals (Jago et al., 2006; Aladro-Gonzalvo et al., 2012), but, due to methodological concerns (e.g., lack of control group, small sample size, lack of a complete methodological description), conclusive results are not yet available. More high-quality studies assessing different physical fitness parameters have been suggested to establish the effects of Pilates (Aladro-Gonzalvo et al., 2012).

This study compares the effects of Pilates and traditional aerobic training (walking) programs on body composition, cardiorespiratory fitness, muscular performance (strength, endurance, and flexibility), and functional tasks for overweight or obese subjects without changes in diet. We hypothesized that Pilates results in similar benefits in cardiorespiratory fitness and body composition and higher benefits in isokinetic muscular strength and functional task outcomes compared to traditional aerobic training

\section{MATERIALS AND METHODS}

\section{Participants}

Female and male adults ranging from 30 to 66 years of age who are overweight or obese were invited to participate in the study. The medical school where the study was performed included almost 5500 staff members and 6000 students, and the volunteers were drawn from this community. Inclusion criteria were: present BMI higher than $25 \mathrm{~kg} / \mathrm{m}^{2}$ and be classified as 
116 insufficiently active or sedentary according to International Physical Activity Questionnaire

117 (IPAQ) (Craig et al. 2003). Exclusion criteria were present neurological, cognitive, orthopedic and

118 respiratory disease. Subjects presenting cardiovascular or endocrine disease which were not

119 controlled were also excluded. In addition, subjects who presented cardiovascular events during

120 the exercise tests were also excluded. From July 2013 until December 2014, 115 volunteers

121 performed a medical exam taking into account a medical history questionnaire and a medical

122 physical exam. The follow-up for the first group of participants began in September 2013 and the

123 last group ended in July 2015. Forty-six volunteers were excluded after the application of the

124 inclusion and the exclusion criteria; the reasons for exclusion were: presence of cardiovascular

125 disease $(n=45)$ and presence of arrhythmia $(n=1)$.

126 Of the 69 remaining participants, 25 were allocated to the control group, since the 127 intervention protocols (Pilates or walking sessions) occurred during their working hours. The 128 remaining 44 participants were randomly assigned by drawing their names written on pieces of 129 paper from an opaque envelope and placing them in one of two experimental groups (Pilates or 130 aerobic). During the entire intervention protocol, 9 volunteers dropped out of participation. One 131 participant from the aerobic group withdrew due to knee pain. Eight participants from the control 132 group did not attend the retest protocol; two of them reported back pain, two had undergone 133 emergency surgeries (appendix and fracture), and four cited personal reasons. A flow diagram illustrating the study design and volunteer participation is presented in Figure 1.

Comorbidities were detected during the medical exam, through self-reporting, with the following description: 3 participants with type 2 diabetes mellitus (Pilates group); 14 participants with hypertension (9 in the Pilates group, 2 in the aerobic group, and 3 in the control group); 18 participants with dyslipidemia (10 in the Pilates group, 3 in the aerobic group, and 5 in the control group), and 11 participants with hypothyroidism (7 in the Pilates group, 2 in the aerobic group, and 2 in the control group). All these presented comorbidities were under treatment and were controlled.

Subjects were informed of the study purpose and protocols, and all provided written informed consent before taking part in the study. The Human Research Ethics Committee of the University approved all experimental procedures (Approval number: 197.451) that complied with the principles outlined in the Declaration of Helsinki. This clinical trial was registered with the 
147 www.ensaiosclinicos.gov.br) and its registration code is RBR-7QNSH6. The authors confirm that 148 all ongoing and related trials for this intervention were duly registered. It is noteworthy that the 149 target sample size described in the clinical trial's registration was 20 participants for each group. 150 To ensure that 20 participants would conclude the study, more than 20 subjects were initially

151

152

153

154

155

156

157

158

159

160

161

162

163

164

165

166

167

168

169

170

171

172

173

174

175

176

177

recruited, and after withdrawals, 17 participants from the control group, 21 from the aerobic group and 22 from the Pilates group participated through to the conclusion of the study.

The age of the participants who completed all phases of experimental protocol $(n=60)$ was $55.9 \pm 6.6 y r s$ (Pilates group), 42.4 $\pm 7.0 y r s$ (aerobic group) and 45.5 $\pm 9.3 y r s$ (control group). Oneway ANOVA (analysis of variance) was used to verify differences in age and height before intervention. The one way ANOVA revealed that Pilates group was older than the other groups $[\mathrm{F}(2,57)=18.521, \mathrm{p}<0.01]$. The height was not significantly different between Pilates $(161 \pm 6 \mathrm{~cm})$, Aerobic $(161 \pm 8 \mathrm{~cm})$ or Control groups $(162 \pm 7)[\mathrm{F}(2,57)=0.25089, \mathrm{p}=0.78]$.

\section{Study design}

This study was a variation of the classic RCT for experimental groups. According to West et al. (2008), classical RCTs rely on the strong adherence of volunteers and, in some situations, very intense encouragement is necessary to guarantee the adherence, creating an unreal situation. In an alternative to this model, volunteers are allowed to choose the treatment they want to receive, rather than being randomly allocated in a group. This option is very interesting situations in which it is impractical or unrealistic to require adherence, and in which the adherence would only be possible through unrealistic encouragement. Considering that some participants could not participate in interventions due to their work activities, we decided to use a randomized encouragement design, since it would be unethical and unrealistic to request the volunteer to resign. Professionals who carried out the tests and measurements were blinded to the treatment conditions.

Since previous studies investigating aerobic training effects had found positive results with eight weeks of training (Ho et al., 2012; Many et al., 2013), participants in the aerobic and Pilates groups followed an experimental protocol for a period of eight weeks, with 60-minute sessions three times per week (totaling 24 sessions). Participants were asked not to initiate any other exercise activity apart from the one included in this study. When a participant missed three or fewer training sessions, the sessions were replaced at the end of the period, but if more than three 
178 sessions were missed, the participant was excluded from the study. Regarding adherence, only

179 three participants needed to replace two exercise sessions at the end of the period, one from the

180 Pilates group and two from the aerobic group. Therefore, by the end of the study, all participants

181 for the aerobic and Pilates groups had completed 24 training sessions.

182 All participants from the three groups underwent tests performed over two consecutive 183 days at the Exercise Physiology Laboratory of the Federal University of São Paulo (São Paulo, 184 Brazil) both before and after the experimental protocol took place. The following assessments were 185 conducted: food intake, cardiorespiratory maximal treadmill test, isokinetic strength testing, body 186 composition and anthropometry, abdominal endurance test, trunk extensor endurance test, 187 flexibility test and functional (stair and chair) tests.

188 As the control group did not follow an experimental protocol, for ethical reasons, control 189 participants were offered the opportunity to participate in the Pilates or Aerobic program, 190 according to the participant's interest, after the conclusion of the study. Those who participated in 191 the experimental protocol were offered the opportunity to participate in the other protocol that they 192 did not take part in initially. Details of the study protocol can be found at 193 http://dx.doi.org/10.17504/protocols.io.p5wdq7e.

\section{Assessments}

\section{Food intake assessment}

Participants were asked to record their food intake for three days (two week days and one weekend day) (Jeon et al., 2011). The participants were asked to write down a description of the foods consumed, quantity per unit or portion sizes, type of preparation, and time of day. The quantitative assessment of the food record was carried out by calculating total energy consumption. An experienced nutritionist performed intake analysis using the Diet Win Professional 2.0 software (Brazil). Participants were instructed to avoid changing their diet habits during the experimental protocol.

\section{Cardiorespiratory maximal treadmill test}

All participants submitted to a cardiorespiratory maximal test on a motorized treadmill (Inbrasport, ATL, Porto Alegre, Brazil) with individualized ramp protocol in order to identify VT, 
209 one minute standing on the treadmill, the participants began the test with 3 minutes of warm-up at

$2103 \mathrm{~km} / \mathrm{h}$ and 0 degree of inclination. From the 4th minute of testing, both speed $(0.5 \mathrm{~km} / \mathrm{h})$ and 211 inclination $(0.5 \%)$ increased every 20 seconds. At the 6 th minute of the test, the participant reached 212 the final speed $(6 \mathrm{~km} / \mathrm{h})$, while the inclination was increased every 20 seconds until the volunteer 213 reached exhaustion. We chose this strategy because overweight/obese population traditionally do 214 not reach high velocities in CPET and due to safety reasons. The same individual protocol for each 215 volunteer was used in the retest.

216 Individual subjective level of exertion was obtained using the Borg scale (Noble et al., 217 1983). In order to determine VT, RCP, and ( $\dot{V} \mathrm{O}_{2}$ max/peak), ventilation and expired gases were 218 measured breath by breath using a metabolic analyzer (Quark, Cosmed, Italy), and all the measured 219 data were considered as a mean of 20 seconds for analysis. Calibration procedures were performed 220 according to the manufacturer's guidelines before each test. VT and RCP were determined 221 separately by two different experienced investigators, according to established criteria (Beaver, 222 Wasserman \& Whipp, 1986). In cases where there was no consensus between the investigators, a 223 third experienced investigator was asked to determine VT and RCP. VT was determined based on 224 the following criteria: inflection in the ventilation curve, increase in the ventilatory equivalent for 225 oxygen without increase in the ventilatory equivalent for dioxide carbon, and increase in partial 226 pressure of exhaled oxygen. RCP was determined based on inflection in the ventilation curve, 227 increase in the ventilatory equivalent for oxygen and in the ventilatory equivalent for dioxide carbon, and decrease in partial pressure of exhaled dioxide carbon (Whipp, Ward \& Wasserman, 1986). Briefly, VT represents the most intense effort (expressed in terms of $\dot{V} \mathrm{O}_{2}$ and/or workload) that can be performed without accumulation of lactate in blood (Whipp, Ward \& Wasserman, 1986).

$\dot{V} \mathrm{O}_{2}$ max was the higher oxygen consumption value at the end of an incremental test, despite an increase in the treadmill speed, featuring the $\dot{V} \mathrm{O}_{2}$ plateau. However, in clinical testing situations, a clear plateau may not be detected. Consequently, $\dot{V} \mathrm{O}_{2}$ peak is often used as an estimate a useful measure of exercise tolerance, and low values indicate poor aerobic physical fitness, and these data also have clinical significance (Howley, 2007). 
After each training session, the aerobic and Pilates groups were asked to provide a rating

241

242

243

244

245

246

247

248

249

250

251

252

253

254

255

256

257

258

259

260

261

262

263

264

265

266

267

268

269

270

of perceived exertion (RPE, Borg scale) adapted by Foster et al. (2001), to calculate the load of each session. Participants were asked about the rate of overall intensity of the training session 30 minutes after the end of the activity. The result was multiplied by the duration of the session, producing an index to monitor the internal training load.

\section{Isokinetic strength testing}

Before the isokinetic testing, the participants performed a 5-min warm-up on a cycle ergometer (Cybex Inc., Ronkonkoma, NY, USA) at a resistance level of 25 watts, followed by low-intensity dynamic stretching exercises for the hamstrings and quadriceps to avoid stretching influence in strength values (Mascarin et al., 2015). Following the warm-up period, participants were placed on the isokinetic dynamometer (Biodex Medical Systems Inc., Shirley, NY, USA) to evaluate the isokinetic concentric strength of both lower limbs in a random order. Peak torque (PT) of knee flexor and extensor muscles (both dominant and non-dominant) in concentric activity was measured. Concentric activity was evaluated at $60 \%$ s (the lowest speed in order to avoid high joint pressure while producing the highest torque values) and $240 \%$ s (an angular speed closer to knee angular speed during the gait) separated by a one-minute rest (Fleury et al., 2011). Low angular speed $\left(60^{\circ} / \mathrm{s}\right)$ was tested first. Participants completed three submaximal trials before each angular speed test to familiarize themselves with the equipment, and five maximal repetitions to test at $60 \%$ s and at $240 \%$ s. The isokinetic dynamometer calibration procedure was performed according to the manufacturer's recommendations.

\section{Body composition and anthropometry}

Body composition was assessed by dual-energy X-ray absorptiometry (DXA, software version 12.3, Lunar DPX, Madison, WI, USA) in order to measure fat-free mass and fat mass. This method has been previously demonstrated to be a safe method with minimum radiation dosage and reliable technique for fat mass (Choi et al., 2015). BMI was calculated by dividing body mass by height squared $\left(\mathrm{kg} / \mathrm{m}^{2}\right)$. A BMI $\geq 25 \mathrm{~kg} / \mathrm{m}^{2}$ indicates overweight, while a BMI $\geq 30 \mathrm{~kg} / \mathrm{m}^{2}$ indicates obesity (WHO, 2012).

Two different points of body circumference were considered: waist circumference (in $\mathrm{cm}$ ) was measured around the midpoint between the lower margin of the last palpable rib and the top 
271 of the iliac crest; hip measurement was taken at the maximum circumference over the buttocks

272 (Reidpath et al., 2013).

273

274

275

276

277

278

279

280

281

282

283

284

285

286

287

288

289

290

291

292

293

294

295

296

297

298

299

300

301

\section{Abdominal endurance test (sit-up test)}

Abdominal endurance was evaluated using the partial trunk flexion test. Participants performed the test lying down with knees flexed at $90^{\circ}$, feet flat on the floor and hands clasped behind the head. The trunk was raised to the point where the shoulders were lifted from the mat. The maximum number of repetitions in one minute was measured (Sarti et al., 1996).

\section{Trunk extensor endurance test}

Subjects underwent the Sorensen test to evaluate isometric endurance of the trunk extensor muscles. The participant lay prone on an examination table with the upper border of the iliac crests aligned with the edges of the table. The lower body was secured by three belts located around the hip, knees, and ankles. The arms were crossed over the chest. Participants were asked to isometrically maintain the upper body in a horizontal position as long as possible. The time to exhaustion was measured in seconds. The test stopped after a maximum of 240 seconds (Demoulin et al., 2006).

\section{Flexibility test}

Flexibility was evaluated using the sit and reach test, which specifically measures the flexibility of the lower back and hamstring muscles. In this test, the participants sit on the floor with lower limbs fully extended, ankles flexed, and feet flat against the box used for the test. The participant must lean slowly and protrude forward as far as possible, sliding their fingers along a ruler affixed to the upper side of the box. After two practice reaches, the participant reaches out and holds the position for two seconds while the distance (in centimeters) is recorded (Wells \& Dillon, 1952).

\section{Functional tests}

Two different tests were performed to assess functional performance in activities of daily living: the stair test and chair test. In the stair test, the subject must climb up and down a set of 12 steps of $16 \mathrm{~cm}$ in height (total of 24 steps) twice. In the chair test, the subject must get up out of 
302

303

304

305

306

307

308

309

310

311

312

313

314

315

316

317

318

319

320

321

322

323

324

325

326

327

328

329

330

331

332

and sit back down into a $50 \mathrm{~cm}$-high chair 10 times. The runtimes for the two tests were recorded in seconds. These tests were adapted from Pescatello et al. (2014).

\section{Interventions}

\section{Pilates protocol}

Participants who were in the Pilates group used a heart rate (HR) monitor (Suunto, Ambit, Finland) during all sessions to verify HR, in order to know at which percentage of maximal HR the volunteers performed the Pilates and in order to compare the mean HR obtained (exercise intensity) of the two experimental protocols. This intervention was performed at a Pilates Studio (CGPA Pilates Studio, São Paulo, Brazil) in groups (5 individuals per group), oriented by the same qualified professional who had graduated in sports and exercise science more than 20 years earlier and had experience in Pilates for 7 years. Each session was composed of: pre-Pilates warm-up exercises (10 min. breathing, shoulder, hip and spine alignment); Pilates (45 min. general conditioning with the traditional Pilates repertoire of exercises for beginners and intermediates, mat Pilates, and the following apparatus/equipment: Reformer, cadillac, chair with adjustable spring resistance; high barrel, and accessories like magic circles and 1-kg dumbbells); and cooldown (5 min. respiratory exercises). All Pilates exercises were performed in a single series of repetitions, and the number of exercises was increased according to the participant's ability to successfully complete the exercise (Table 1). When necessary, exercises were adapted for obese and overweight body dimensions. An example of adaptation is in the Foot Work exercise on the Reformer. The original position of the exercise would be lying down on the Reformer, however some volunteers did not feel comfortable. A modification was made to elevate the trunk to the recline position, with the help of a wooden board leaned against the shoulder guard. In this way the volunteers could do the exercise comfortably, with no suffering.

\section{Aerobic training protocol}

After cardiorespiratory maximal test (see details below), ventilatory threshold (VT) was determined and each volunteer performed the walking program in the HR corresponding to the VT. This walking intensity is considered safe to prevent orthopedic injuries in participants with obesity (McQueen, 2009). Each section of the program lasted 60 minutes and was performed 3 times per week for 8 weeks. In the first 10 minutes of each section, volunteers performed warm- 
333

334

335

336

337

338

339

340

341

342

343

344

345

346

347

348

349

350

351

352

353

354

355

356

357

358

359

360

361

362

363

up exercises. After the warm-up, volunteers walked at an intensity sufficient to maintain the HR in corresponding values to VT intensity for the next 40 minutes, and in the last 10 minutes they performed cooldown exercises. All participants used an HR monitor (Suunto, Ambit, Finland) for monitoring HR during all training sessions, and sessions were supervised at all times by a coach to ensure the HR had been maintained at the target value. The sessions were performed at the Olympic Training and Research Center (Centro Olímpico de Treinamento e Pesquisa; COTP, São Paulo, Brazil), in groups (5 individuals per group).

\section{Statistical analyses}

Statistical analyses were performed using the Statistica software (Statsoft, Inc., version 6.0 for Windows, USA). Data were expressed as mean \pm standard deviation (SD). Variable distribution was tested by the Kolmogorov-Smirnov test, and variability by the Levene test. The sample size was planned to identify a difference between training groups of at least $2 \%$ of fat mass in a relatively homogeneous group of healthy adults. To this end, we used data from a pilot study. The target sample size was 20 participants in each group (alpha 0.05 and power 0.80 ). After data collection, a two-way ANOVA for time vs. group of fat mass (\%) and the power observed (alpha $=0.05$ ) yielded the interaction effect of 0.87 ; therefore, we conclude that the sample size was sufficient for subsequent analysis.

To verify the effects of the intervention programs, a two-way ANCOVA, conducted by using age as a covariate, was used to assess the groups (control vs. aerobic vs. Pilates) and the time (pre- vs. post-training) differences for the following measures: anthropometric and body composition; waist and hip circumferences; abdominal, trunk, chair, stair and flexibility tests; and isokinetic and cardiorespiratory measurements. Newman-Keuls post hoc test procedures were used to identify specific differences. In the absence of interactions, only main effects were analyzed. Where age presented no significant difference for the variable analyzed in ANCOVA test, a two-way ANOVA was used.

In order to compare the HR and RPE reached during the sessions between the two intervention groups we used an unpaired Student's $t$ test. Statistical significance was set at an $\alpha$ of 0.05 for all statistical procedures.

Calculation of effect size $(d)$ was adopted in addition to the traditional statistical approaches. The measures of the effect size for changes in outcome were calculated by dividing 
364 the mean difference by the SD of the pre-training measurement. By calculating effect sizes, the 365 magnitude of any change was judged according to the following criteria: $d=0.2$ was considered a 366 "small" effect size; $d=0.5$ represented a "medium" effect size; and $d=0.8$ was a "large" effect 367 size (Cohen, 1988). Percent changes and 90\% confidence intervals were also calculated..

368

369

370

371

372

373

374

375

376

377

378

379

380

381

382

383

384

385

386

387

388

389

390

391

392

393

394

\section{RESULTS}

All volunteers performed 24 exercise sessions. The two-way ANOVA (group vs. time) revealed that there were no significant differences (pre- vs. post-training) in calorie intake for all groups $[\mathrm{F}(2,57)=0.02744, \mathrm{p}=0.97)]$. The target HR training for the aerobic group was $123 \pm 11$ bpm, which represents about $78.0 \pm 7.3 \%$ of the maximal HR $(160 \pm 16 \mathrm{bpm})$ reached during the cardiorespiratory maximal treadmill test. The Pilates group reached an HR training of $86 \pm 6 \mathrm{bpm}$, which represents about $62.7 \pm 13.2 \%$ of the maximal HR reached in the cardiopulmonary treadmill test. Thus, HR during training was significantly higher $(\Delta \%=+42.2 \%)$ in the aerobic group than in the Pilates group $(\mathrm{p}<0.0001$; see Table 2). Moreover, the internal training load was also higher $(\Delta \%=+31.3 \%)$ in the aerobic group than in the Pilates group $(\mathrm{p}=0.04$; see Table 2$)$.

Two-way ANCOVA (group vs. time), using age as a covariate, revealed that the age presented a significant effect for abdominal strength test, flexibility test, stair test, peak torque for dominant and non-dominant extensor muscles at $240 \%$ s and HR assessed at VT, RCP and maximal exercise. Therefore, the results presented for these variables were from ANCOVA using age as a covariate. Results presented for the other variables (BMI, total body mass, fat mass, fat free mass, waist and hip circumference, trunk endurance test, chair test, peak torque for extensor and flexors muscles at $60 \%$ s and $240 \%$ s and for flexors at 240/s and $\dot{V} \mathrm{O}_{2}$ at VT, RCP, or maximal exercise) were from ANOVA (group vs. time), once age presented no significant difference in the analysis of these variables.

There were no significant differences in body composition, anthropometric measurements, and functional tasks among the three groups at baseline (Table 3), except for the abdominal endurance test $[\mathrm{F}(2,57)=3.69, \mathrm{p}=0.031]$. The aerobic group had higher values than both the control group $(\mathrm{p}=0.02)$ and the Pilates group $(\mathrm{p}=0.01$; see Table 3$)$.

As expected, after an 8-week period, the control group showed no differences in any of the outcome variables., except for abdominal test performance with higher values after the experimental period $(\mathrm{p}=0.02, d=0.57$ and $\Delta \%=+9.3 \pm 14.8 \%)$. Total body mass did not change 
395 in any of the groups and despite the significant improvement, in fat mass $(\Delta \%=-3.7 \pm 5.2 \%)$ and 396 lean mass $(\Delta \%=+3.2 \pm 5.0 \%)$ in the Pilates group, its effect was classified as small $(d=0.19)$. 397 There was no difference in body composition between groups after the intervention period 398 ( $\mathrm{p}>0.05)$. As expected, the Pilates and aerobic groups showed significant improvements in waist 399 circumference $(p=0.0009, p=0.0001$, respectively), hip circumference $(p=0.0001, p=0.001$, 400 respectively), abdominal test $(p=0.0001, p=0.003$, respectively), and chair test $(p=0.0001$, 401 $\mathrm{p}=0.005$, respectively) and stair test $(\mathrm{p}=0.003, \mathrm{p}=0.046$, respectively). Trunk test and flexibility test improved only in the Pilates group $(\mathrm{p}=0.0003$ and $\mathrm{p}=0.0001$, respectively; see Table 3 ).

As both the Pilates and aerobic groups showed significant improvements in some outcome variables after the intervention, the effect size was also measured and the difference between pre405 and post-tests was also expressed as perceptual difference (Table 3). The effect size and perceptual 406 difference of the Pilates and aerobic training in waist $(d=0.27, \Delta \%=-2.6 \pm 2.9 \%$ and $d=0.37$, $\Delta \%=-3.3 \pm 3.7 \%$, respectively) and hip circumference $(d=0.17, \Delta \%=-1.5 \pm 1.6 \%$ and $d=0.14$, $\Delta \%=-0.8 \pm 1.0 \%$, respectively) were classified as medium for waist values and small for hip values. There was also no significant difference between groups after intervention $(\mathrm{p}>0.05)$. Despite a significant improvement in the abdominal test in all three groups $(d=1.69$, $\Delta \%=25.6 \pm 13.5 ; d=0.95, \Delta \%=11.0 \pm 16.7 \%$ and $d=0.58, \Delta \%=9.3 \pm 14.8 \%$ for Pilates, aerobic and control groups, respectively), the effect size and perceptual difference for the Pilates group were greater than the effect size and perceptual difference for the aerobic group, which also were greater than these variables for the control group. Moreover, after the intervention period, the control group showed significantly lower values for the abdominal test than the Pilates $(\mathrm{p}=0.005)$ and aerobic groups $(\mathrm{p}=0.004)$.

Results of the two evaluated functional tasks differed across the groups. On the chair test $[\mathrm{F}(2,57)=8.49, \mathrm{p}=0.00]$, the improvement observed in the Pilates group $(\Delta \%=-26.9 \pm 27.9 \%$, $d=1.48, \mathrm{p}=0.0001)$ was greater than that seen in the aerobic group $(\Delta \%=-11.3 \pm 16.5 \%, \mathrm{p}=$ $0.005, d=0.75)$. Moreover, only the Pilates group showed a significant improvement performance in the chair test than the control group $(\mathrm{p}=0.02, \Delta \%=30.0 \pm 27.9 \%)$ after the intervention period. In the same direction, the stair test $[\mathrm{F}(2,57)=4.00, \mathrm{p}=0.024]$. The improvement observed in the Pilates group was also greater than that seen in the aerobic group $(d=0.78, \Delta \%=9.8 \pm 7.9 \%$ and $d=0.41, \Delta \%=7.4 \pm 10.5 \%$, respectively). However, there were no significant differences between groups after the intervention period. 
Isokinetic PT for dominant $(\mathrm{p}=0.02)$ and non-dominant $(\mathrm{p}=0.02)$ extensor muscles

$427\left(60^{\circ} / \mathrm{s}\right)$ were significantly lower for the Pilates group than for controls at baseline. However, after 428 the 8-week period, neither isokinetic PT of flexor nor for extensor muscles in both dominant and 429 non-dominant limbs presented significant differences (pre- vs. post-intervention) in any of the 430 three groups at any angular speed tested $(60 \% \mathrm{~s}$ and $240 \% \mathrm{~s}$; see Table 4$)$.

431 Regarding cardiorespiratory fitness prior to the intervention period at baseline, there were 432 no significant differences between the three groups in $\dot{V} \mathrm{O}_{2}$ values at VT, RCP, or max values (p > 433 0.05). The Pilates group presented lower HR values than the aerobic $(\mathrm{p}=0.0002)$ and control 434 groups $(p=0.0002)$ in VT, and than the aerobic $(p=0.004)$ and control $(p=0.005)$ groups in RCP, 435 and lower values than the aerobic $(p=0.03)$ and control $(p=0.001)$ group in maximal effort. After 436 the training period, the Pilates group presented a significant increase in $\dot{V} \mathrm{O}_{2}$ and in HR reached at 437 VT $(p=0.001$ and $p=0.0005$, respectively), RCP $(p=0.01$ and $p=0.01$, respectively) and at 438 maximum effort $(p=0.01$ and $p=0.003$, respectively) (Table 5). The control group presented 439 significant different HR in maximal effort after and before the intervention period $(\mathrm{p}=0.02)$. The 440 aerobic group presented no significant difference in any cardiorespiratory variables measured 441 (Table 5). Despite the significant increase in $\dot{V} \mathrm{O}_{2}$ at VT $(\mathrm{p}=0.001, d=0.60, \Delta \%=11.6 \pm 17.6 \%)$, $\operatorname{RCP}(\mathrm{p}=0.01, d=0.48, \Delta \%=7.9 \pm 19.5 \%)$ and maximum values $(\mathrm{p}=0.01, d=0.33$, $\Delta \%=19.9 \pm 15.5 \%)$ observed only in the Pilates group, after the experimental period there were no differences between the groups.

\section{DISCUSSION}

This study compared the impact of Pilates and aerobic training on cardiorespiratory fitness, isokinetic muscular strength, body composition, flexibility, trunk and abdominal endurance, and functional task outcomes for overweight/obese individuals. Our main findings were that BMI, lean mass, fat mass, waist circumference, hip circumference, abdominal and trunk strength tests, chair and stair test, flexibility and $\dot{V} \mathrm{O}_{2}$ assessed at VT, RCP and at maximum effort improved in Pilates group. Conversely, only waist and hip circumference, abdominal strength test, and chair and stair test improved in aerobic training group. Abdominal strength test was lower in control group than in both intervention groups in post-tests and chair test were lower in control than in Pilates group 455 in post-tests. 


\section{Body composition effects}

458

459

460

461

462

463

464

465

466

467

468

469

470

471

472

473

474

475

476

477

478

479

480

481

482

483

484

485

486

487

Traditionally, body mass reduction by decreasing fat mass is the main goal in obesity treatment, and several studies have investigated the effects of exercise for that purpose, although the importance of physical activity for weight loss assessed by body weight or BMI is controversial (Pedersen \& Saltin, 2015). The aerobic exercise protocol employed in the present study promoted no difference in body composition. Cheema et al. (2015) also failed to find a significant loss of fat mass percentage after an aerobic program performed at moderate intensity for 12 weeks. Probably, the amount of exercise (volume and/or intensity) necessary to produce weight loss needed to be greater than the one implemented in our study, and a restrictive diet would also be necessary for body mass loss.

Changes induced by the Pilates were greater than those promoted by aerobic training, as the Pilates group presented a significant increase in lean mass and decrease in fat mass. These results suggest that Pilates may be an interesting alternative to traditional aerobic training in order to improve body composition (Jago et al., 2006; Rogers \& Gibson, 2009; Şavkin \& Aslan, 2017). Conversely, due to the small effect size, the results should be viewed with caution. Moreover, there were no differences between groups after the intervention period. Previous studies also show effective results for Pilates in reducing fat mass and increasing fat free mass (Rogers \& Gibson, 2009; Şavkin \& Aslan, 2017). Therefore, despite the small effect size observed in our study after the Pilates, our results are in line with the literature. Conversely, these results are not a consensus. Segal, Hein \& Bastford (2004) showed no significant difference in body composition after Pilates. Methodological differences may be responsible for these conflicting results; different indirect methods - which tend to be inaccurate - used to assess body composition (Rogers \& Gibson, 2009); a lack of information about the instructors' certification (Segal, Hein \& Basford, 2004; Rogers \& Gibson, 2009); and significant differences in the protocol used (Pilates mats or apparatus, sessions per week and program duration); associated with a lack of diet control (Segal, Hein \& Basford, 2004) hamper definitive conclusions on this topic. Considering that DXA, which has been previously demonstrated to be reliable (Choi et al., 2015) was used in our study to measure body composition, it can be concluded that Pilates may be considered an alternative exercise program for improving body composition.

\section{Functional tasks tests}


Both the aerobic and Pilates groups showed significant improvement on both tests, unlike

489

490

491

492

493

494

495

496

497

498

499

500

501

502

503

504

505

506

507

508

509

510

511

512

513

514

515

516

517

518

the control group. However, the improvements on the chair and stair tests in the Pilates group were greater (i.e., had a higher effect size) than in the aerobic group. Moreover, only the Pilates group presented significantly better results in the chair test than the control group after the intervention. This improvement in functional tasks presented in both experimental groups is of fundamental importance for independent living and mobility (Miller 2008).

\section{Abdominal and trunk strength test and flexibility test}

The abdominal and trunk strength tests performed in this study have been found to be reliable field tests (Sparling, Millard-Stafford \& Snow, 1997; Demoulin et al., 2006). Moreover, sit and reach test has also been found to be reliable (Bozic et al., 2010). All three groups had higher values for the abdominal test after the 8-week intervention, suggesting that there was a learning effect on this test demonstrated by the improvement in the control group. Notwithstanding, both the intervention groups presented significantly higher results in the abdominal test after the intervention period than the control group. Demonstrating that both the aerobic and Pilates interventions were effective to improve abdominal strength. It is important to notice that the Pilates was more effective to improve abdominal muscle strength, demonstrated by the higher effect size value. In the same direction, trunk extensor muscle endurance showed a significant improvement only in the Pilates group, in the same way as flexibility. In general, these results were expected, since the Pilates method focuses on these muscles through posture and core exercises (Şavkin \& Aslan, 2017). Although the effect size of this improvement was classified as large, after the intervention period there were no significant differences between groups. The individuals from each group were quite different and the SD of the measurements was very high; this may be the reason for the absence of significant difference between groups after the intervention, despite the improvement observed only in the Pilates group. We therefore conclude that for functional tasks (chair and stair tests), trunk and abdominal endurance tests, both training programs should be considered, but the Pilates can produce better results.

\section{Isokinetic strength test}

Isokinetic dynamometry is a method commonly used to assess muscle performance, both in research and in clinical practice (Granata, Abel \& Damiano, 2000; Fleury et al., 2011; Andrade 
519 et al., 2013). As has been previously demonstrated, isokinetic dynamometry is a reliable and

520 sensitive instrument to evaluate therapeutic intervention outcomes, including in young or elderly

521 people (Capranica et al., 1998; Eitzen, Hakestad \& Risberg, 2012; Andrade et al., 2013).

522 Isokinetic muscular strength, mainly in the Pilates group, was expected to show a

523 significant improvement, but there were no changes in isokinetic PT for either muscular group 524 assessed (knee flexor and extensor muscles) at 60\% and $240 \%$ s. Since Pilates exercises are not 525 isokinetic exercises, the mode of contraction and the angular speed are different, and this lack of 526 specificity of the evaluation (isokinetic dynamometer) may contribute to unexpected results. 527 Another possible explanation for the absence of difference in isokinetic strength results after the 528 intervention in the Pilates group may be related to the lower power of isokinetic analysis. For 529 flexor and extensor PT values, the observed power ranged from 0.06 to 0.43 . Lower power means 530 that there is a high probability of concluding there is no effect when one actually exists. In other 531 words, the number of subjects in this study might be not sufficient to ensure (to a particular degree 532 of certainty) that the interventions produced no effects on muscular isokinetic strength.

533

\section{Cardiorespiratory maximal treadmill test}

535 In the present study, the Pilates group showed a significant increase in $\dot{V} \mathrm{O}_{2}$ max after the 536 intervention, which did not occur in the aerobic group, although the Pilates intervention was less 537 intense than the walking intervention (internal training load $192 \pm 69$ vs. $252 \pm 106$ ). The lack of $\dot{V}$ $538 \mathrm{O}_{2}$ max improvement after aerobic training was in accordance with previous data (Shinkai et al., 539 1994; Fiorilli et al., 2017). Shinkai et al. (1994) studied the effects on $\dot{V} \mathrm{O}_{2}$ max of a 12-week 540 aerobic program plus voluntary food restriction for mildly obese middle-age women. The authors 541 observed no change in absolute $\dot{V} \mathrm{O}_{2} \mathrm{max} /$ peak values (L/min), and a significant increase only in 542 relative $\dot{V} \mathrm{O}_{2} \mathrm{max} /$ peak values $(\mathrm{mL} / \mathrm{kg} / \mathrm{min})$ after the program, which could be attributed to the 543 significant body mass loss observed, instead of a real $\dot{V} \mathrm{O}_{2}$ max improvement. Fiorilli et al. (2017) 544 also studied the effects on $\dot{V} \mathrm{O}_{2}$ max of an aerobic or resistance program. Interestingly, their results 545 showed higher $\dot{V} \mathrm{O}_{2}$ max values for the resistance-training group than for the aerobic training group.

With regards to $\dot{V} \mathrm{O}_{2}$ max, this variable can be derived from the Fick equation: $\dot{V} O_{2}$ $547 \max =\mathrm{COmax} x(\mathrm{Ca}-\mathrm{C} \overline{\mathrm{v}}) \mathrm{O}_{2} \max$. Therefore, $\dot{\mathrm{V}} \mathrm{O}_{2} \max$ depends on the maximal $\mathrm{CO}$ (cardiac 548 output) and the maximal $(\mathrm{Ca}-\mathrm{C} \bar{v}) \mathrm{O}_{2}$ (difference between oxygen concentration taken from 549 arterial and mixed venous blood). In most situations, $(\mathrm{Ca}-\mathrm{C} \overline{\boldsymbol{v}}) \mathrm{O}_{2}$ does not vary much between 
550 individuals, so the $\dot{V} \mathrm{O}_{2} \mathrm{max}$ is usually limited by the maximal CO (Bassett \& Howley, 2000); 551 however, in some special situations, such as detraining, very sedentary people or muscular 552 dystrophy, it may be limited by a low maximal $(\mathrm{Ca}-\mathrm{C} \bar{v}) \mathrm{O}_{2}$, resulting from a low muscular mass 553 capacity.

$554 \quad$ It is probable that the $\dot{V} \mathrm{O}_{2} \mathrm{max} /$ peak improved in the Pilates group as a consequence of a 555 higher maximal $\mathrm{CO}$ resulting from the higher maximal HR reached at the end of the 556 cardiopulmonary test. Moreover, as indicated above, in detraining or very sedentary people, the $\dot{V}$ $557 \mathrm{O}_{2}$ max may be limited by a low maximal $(\mathrm{Ca}-\mathrm{C} \overline{\boldsymbol{v}}) \mathrm{O}_{2}$. Considering that the Pilates group exercises 558 are focused on muscular conditioning, it is possible that the increase in the $\dot{V} \mathrm{O}_{2} \mathrm{max} /$ peak resulted 559 from the observed muscular mass improvement after the program, too. However, it was not 560 possible to state that the aerobic capacity of the muscular mass has increased. The aerobic group 561 failed to show an improvement in the $\dot{V} \mathrm{O}_{2} \mathrm{max} /$ peak after the training period. During the walking 562 session, the HR mean was $123 \pm 11 \mathrm{bpm}$ (HR corresponding to the VT), which represents about $56378 \%$ of the maximal HR for this group, which is in accordance with aerobic program 564 recommendations (training intensity greater than $60 \%$ of the HRmax, with 3-5 sessions/week for $56530 \mathrm{~min} / \mathrm{session})$. Although moderate aerobic exercise has been extensively suggested for obese 566 people (Pedersen \& Saltin, 2015; Burgess et al., 2017; Mabire et al., 2017), our data were in line 567 with other previous literature data (Cheema et al., 2015) which demonstrated that moderate 568 walking performed for 3 months (4 times a week) was not effective to improve aerobic fitness in 569 overweight and obese adults. It is possible that an even greater effort intensity or training period 570 is necessary to produce an increase of maximal $\mathrm{CO}$ and/or maximal $(\mathrm{Ca}-\mathrm{C} \bar{v}) \mathrm{O}_{2}$ resulting in 571 greater $\dot{V} \mathrm{O}_{2} \mathrm{max} /$ peak. Other studies that used high-intensity interval training showed significant 572 improvement in the $\dot{V} \mathrm{O}_{2} \max /$ peak (Cheema et al., 2015; Ruffino et al., 2017), so exercise 573 intensity may be crucial to improve the $\dot{V} \mathrm{O}_{2}$ max/peak.

574 Although there was no significant difference between groups after the intervention period, 575 the improvement in $\dot{V} \mathrm{O}_{2} \mathrm{max} /$ peak in the Pilates group was classified as medium, and considering 576 the strong association between an increase in the $\dot{V} \mathrm{O}_{2} \mathrm{max} /$ peak and reduced all-cause and 577 cardiovascular disease mortality (Lee et al., 2011; Barlow et al., 2012), this is a meaningful benefit 578 of Pilates.

579 VT is another aerobic index that has great practical application. Besides VT, prolonged 580 exercise leads to progressive metabolic acidosis and exhaustion. VT depends on peripheral 
581 circulatory factors, specifically on the oxidative capacity of the contracting muscles (Weltman, 582 1995). In this study, the $\dot{V} \mathrm{O}_{2}$ in the VT increased significantly only in the Pilates group $(d=0.6)$. 583 Muscle conditioning is one of the objectives of the Pilates. Possibly better muscle conditioning, 584 characterized by improved peripheral capillarization and muscle oxidative capacity, were 585 responsible for the $\dot{V} \mathrm{O}_{2}$ improvement measured at the VT. In order to better understand the $\dot{V}$ $586 \mathrm{O}_{2}$ max/peak, VT, and RCP improvement in the Pilates group, future studies should evaluate 587 muscular oxidative capacity. In addition, further studies, aiming to investigate the Pilates and 588 walking training effects on more general health parameters, such as the risk of morbidity and 589 mortality, or on muscular oxidative capacity should be developed.

590

591

592

593

594

595

596

597

598

599

600

601

602

603

604

605

606

607

608

609

610

\section{Strengths and limitations of this study}

The external validity of the study was guaranteed through an extensive process of volunteer recruitment (social networks and within the medical school) and through the randomization process that divided the volunteers between the two intervention groups. One should, however, consider the cost of a Pilates practice program in a studio. This aspect should be taken into consideration in the appointment of a physical health program for individuals who are overweight/obese.

A possible bias of this study is the inclusion of both men and women. However, an attenuating factor is that both intervention groups contained the same number of men.

Another possible bias is the lack of randomization between control and experimental groups. Twenty-five volunteers asked to participate in the control group, because the intervention protocol (Pilates or walking sessions) was during their working hours. If a random order were used, very intense encouragement would be necessary to guarantee adherence, creating an unreal situation. In order to avoid this bias, these volunteers were allocated to the control group. Choosing for non-randomization between the control and experimental groups could have created a bias as a result of the lack of homogeneity of the baseline data, which, fortunately, did not occur; there was no significant difference in baseline data for muscular performance (strength, endurance, and flexibility), body composition, or functional tasks outcomes.

The same intervention period of 8 weeks was performed for both Aerobic and Pilates groups. It was chosen as it was important that the two groups were submitted to the same training 
611 volume. Considering that the aerobic training is usually performed for 12 weeks in previous

612 studies, perhaps other outcomes can be found with longer intervention periods.

613

614 CONCLUSION

615 To our knowledge, this is the first study comparing Pilates with traditionally aerobic 616 exercise for overweight or obese subjects. Pilates should be considered as an interesting alternative 617 physical fitness program for individuals who are overweight or obese, since it can lead to 618 significant improvements in body composition, general strength (trunk and abdominal), flexibility, 619 ability to accomplish functional tasks, and cardiorespiratory fitness.

620

621

\section{ACKNOWLEDGMENTS}

622 We would like to thank all of the participants who volunteered their time to participate in 623 the study, the Olympic Training and Research Center (Centro Olímpico de Treinamento e 624 Pesquisa, COTP, São Paulo, Brazil), and the CGPA Pilates Studio (São Paulo, Brazil). Ricardo 625 Borges Viana had a fellowship from the Coordenação de Aperfeiçoamento de Pessoal de Ensino 626 Superior (CAPES/ - Coordination for the Improvement of Higher Education Personnel, Brazil).

627

628

629

\section{REFERENCES}

630

Aladro-Gonzalvo AR., Machado-Díaz M., Moncada-Jiménez J., Hernández-Elizondo J., Araya-

631

632

633

634

635

636

637

638

639

Vargas G. 2012. The effect of Pilates exercises on body composition: A systematic review. Journal of Bodywork and Movement Therapies 16:109-114. DOI: 10.1016/j.jbmt.2011.06.001.

Andrade M dos S., de Lira CAB., Vancini RL., de Almeida AA., Benedito-Silva AA., da Silva AC. 2013. Profiling the isokinetic shoulder rotator muscle strength in 13- to 36-year-old male and female handball players. Physical Therapy in Sport 14:246-252. DOI: 10.1016/j.ptsp.2012.12.002.

Barlow CE., Defina LF., Radford NB., Berry JD., Cooper KH., Haskell WL., Jones LW., Lakoski SG. 2012. Cardiorespiratory fitness and long-term survival in "low-risk" adults. 640 Journal of the American Heart Association 1:e001354. DOI: 10.1161/JAHA.112.001354.

641

Barry VW., Baruth M., Beets MW., Durstine JL., Liu J., Blair SN. 2014. Fitness vs. fatness on 
642

643

644

645

646

647

648

649

650

651

652

653

654

655

656

657

658

659

660

661

662

663

664

665

666

667

668

669

670

671

672

all-cause mortality: A meta-analysis. Progress in Cardiovascular Diseases 56:382-390.

DOI: 10.1016/j.pcad.2013.09.002.

Barry BK., Carson RG. 2004. The consequences of resistance training for movement control in older adults. The Journals of Gerontology. Series A, Biological Sciences and Medical Sciences 59:730-754. DOI: 10.1093/gerona/59.7.M730.

Bassett DR., Howley ET. 2000. Limiting factors for maximum oxygen uptake and determinants of endurance performance. Medicine and Science in Sports and Exercise 32:70-84. DOI: 10.1097/00005768-200001000-00012.

Beaver WL., Wasserman K., Whipp BJ. 1986. A new method for detecting anaerobic threshold by gas exchange. Journal of Applied Physiology 60:2020-2027. DOI: 10.1152/jappl.1986.60.6.2020.

Bozic PR., Pazin NR., Berjan BB., Planic NM., Cuk ID. 2010. Evaluation of the field tests of flexibility of the lower extremity: Reliability and the concurrent and factorial validity. Journal of Strength and Conditioning Research 24:2523-2531. DOI: 10.1519/JSC.0b013e3181def5e4.

Burgess E., Hassmén P., Welvaert M., Pumpa KL. 2017. Behavioural treatment strategies improve adherence to lifestyle intervention programmes in adults with obesity: a systematic review and meta-analysis. Clinical Obesity 7:105-114. DOI: 10.1111/cob.12180.

Capranica L., Battenti M., Demarie S., Figura F. 1998 Reliability of isokinetic knee extension and flexion strength testing in elderly women. Journal of Sports Medicine and Physical Fitness 38:169-176. DOI: 10.1590/S1413-35552012005000074.

Cheema BS., Davies TB., Stewart M., Papalia S., Atlantis E. 2015. The feasibility and effectiveness of high-intensity boxing training versus moderate-intensity brisk walking in adults with abdominal obesity: a pilot study. BMC Sports Science, Medicine and Rehabilitation 7:3. DOI: 10.1186/2052-1847-7-3.

Chodzko-Zajko WJ., Proctor DN., Fiatarone Singh MA., Minson CT., Nigg CR., Salem GJ., Skinner JS., Skinner JS. 2009. American College of Sports Medicine position stand. Exercise and physical activity for older adults. Medicine \& Science in Sports \& Exercise 41:1510-1530. DOI: 10.1249/MSS.0b013e3181a0c95c.

Cohen J. 1988. Statistical power analysis for the behavioral sciences statistical power analysis for the behavioral sciences. Hillsdale-New Jersey: Lawrence Erlbaum Associates. 
673 Choi YJ., Seo YK., Lee EJ., Chung YS. 2015. Quantification of visceral fat using dual-energy x674 ray absorptiometry and its reliability according to the amount of visceral fat in Korean 675 adults. Journal of Clinical Densitometry 18:192-197. DOI: 10.1016/j.jocd.2015.02.001. 676 Craig CL., Marshall AL., Sjostrom M., Bauman AE., Booth ML., Ainsworth BE., Pratt 677 M., Ekelund U., Yngve A., Sallis JF., Oja P. 2003. International Physical Activity 678 Questionnaire: 12-Country Reliability and Validity. Medicine \& Science in Sports \& 679 Exercise 35:1381-1395. DOI: 10.1249/01.MSS.0000078924.61453.FB.

680 Demoulin C., Vanderthommen M., Duysens C., Crielaard J-M. 2006. Spinal muscle evaluation 681 using the Sorensen test: a critical appraisal of the literature. Joint Bone Spine 73:43-50. 682 DOI: 10.1016/j.jbspin.2004.08.002.

683 Eitzen I., Hakestad KA., Risberg MA. 2012. Inter- and intrarater reliability of isokinetic thigh 684 muscle strength tests in postmenopausal women with osteopenia. Archives of Physical 685 Medicine and Rehabilitation 93:420-427. DOI: 10.1016/j.apmr.2011.10.001.

686 Fiorilli G., Iuliano E., Aquino G., Campanella E., Tsopani D., Di Costanzo A., Calcagno G., di 687 688 689 690 691 692 693 694 695 696 697 698 699 700 701 702 703 Cagno A. 2017. Different consecutive training protocols to design an intervention program for overweight youth: a controlled study. Diabetes, Metabolic Syndrome and Obesity 10:37-45. DOI: 10.2147/DMSO.S122110.

Fleury AM., Silva AC da., Pochini A., Ejnisman B., Lira CAB de., Andrade M dos S. 2011. Isokinetic muscle assessment after treatment of pectoralis major muscle rupture using surgical or non-surgical procedures. Clinics 66:313-320. DOI: 10.1590/S180759322011000200022.

Foster C., Florhaug JA., Franklin J., Gottschall L., Hrovatin LA., Parker S., Doleshal P., Dodge C. 2001. A new approach to monitoring exercise training. Journal of Strength and Conditioning Research 15:109-115. DOI: 10.1519/00124278-200102000-00019.

Granata KP., Abel MF., Damiano DL. 2000. Joint angular velocity in spastic gait and the influence of muscle-tendon lengthening. The Journal of Bone and Joint Surgery. American Volume 82:174-186. DOI: 10.2106/00004623-200002000-00003.

Harber MP., Konopka AR., Undem MK., Hinkley JM., Minchev K., Kaminsky LA., Trappe TA., Trappe S. 2012. Aerobic exercise training induces skeletal muscle hypertrophy and agedependent adaptations in myofiber function in young and older men. Journal of Applied Physiology 113:1495-1504. DOI: 10.1152/japplphysiol.00786.2012. 
704 Ho SS., Radavelli-Bagatini S., Dhaliwal SS., Hills AP., Pal S. 2012. Resistance, aerobic, and 705 combination training on vascular function in overweight and obese adults. The Journal of 706 Clinical Hypertension 14:848-854. DOI: 10.1111/j.1751-7176.2012.00700.x.

707 708 709

Howley ET. 2007. VO2max and the plateau-needed or not? Medicine and Science in Sports and Exercise 39:101-2. DOI: 10.1249/mss.0b013e31802dc897.

Jago R., Jonker ML., Missaghian M., Baranowski T. 2006. Effect of 4 weeks of Pilates on the body composition of young girls. Preventive Medicine 42:177-180. DOI: 10.1016/j.ypmed.2005.11.010.

Jeon KJ., Lee O., Kim H-K., Han SN. 2011. Comparison of the dietary intake and clinical characteristics of obese and normal weight adults. Nutrition Research and Practice 5:329336. DOI: 10.4162/nrp.2011.5.4.329.

Küçükçakır N., Altan L., Korkmaz N. 2013. Effects of Pilates exercises on pain, functional status and quality of life in women with postmenopausal osteoporosis. Journal of Bodywork and Movement Therapies 17:204-211. DOI: 10.1016/j.jbmt.2012.07.003.

Lee D -c., Sui X., Artero EG., Lee I-M., Church TS., McAuley PA., Stanford FC., Kohl HW., Blair SN. 2011. Long-term effects of changes in cardiorespiratory fitness and body mass index on all-cause and cardiovascular disease mortality in men: the aerobics center longitudinal study. Circulation 124:2483-2490. DOI: 10.1161/CIRCULATIONAHA.111.038422.

Mabire L., Mani R., Liu L., Mulligan H., Baxter D. 2017. The influence of age, sex and body mass index on the effectiveness of brisk walking for obesity management in adults: A systematic review and meta-analysis. Journal of Physical Activity and Health 14:389-407. DOI: 10.1123/jpah.2016-0064.

Many G., Hurtado M-E., Tanner C., Houmard J., Gordish-Dressman H., Park J-J., Uwaifo G., Kraus W., Hagberg J., Hoffman E. 2013. Moderate-intensity aerobic training program improves insulin sensitivity and inflammatory markers in a pilot study of morbidly obese minority teens. Pediatric Exercise Science 25:12-26. DOI: 10.1123/pes.25.1.12.

Mascarin NC., Vancini RL., Lira CAB., Andrade MS. 2015. Stretch-induced reductions in throwing performance are attenuated by warm-up before exercise. Journal of Strength and Conditioning Research 29:1393-1398. DOI: 10.1519/JSC.0000000000000752.

McQueen MA. 2009. Exercise Aspects of Obesity Treatment. The Ochsner Journal 9:140-143. 
735 Miller SL., Wolfe RR. 2008. The danger of weight loss in the elderly. Journal of Nutrition,

736

737

738

739

740

741

742

743

744

745

746

747

748

749

750

751

752

753

754

755

756

757

758

759

760

761

762

763

764

765 Health \& Aging 12:487-491. DOI: 10.1007/BF02982710.

Muscolino JE., Cipriani S. 2004. Pilates and the "powerhouse"-I. Journal of Bodywork and Movement Therapies 8:15-24. DOI: 10.1016/S1360-8592(03)00057-3.

Noble BJ., Borg GA., Jacobs I., Ceci R., Kaiser P. 1983. A category-ratio perceived exertion scale: relationship to blood and muscle lactates and heart rate. Medicine and Science in Sports and Exercise 15:523-528. DOI: 10.1249/00005768-198315060-00015.

de Oliveira Francisco C., de Almeida Fagundes A., Gorges B. 2015. Effects of Pilates method in elderly people: Systematic review of randomized controlled trials. Journal of Bodywork and Movement Therapies 19:500-508. DOI: 10.1016/j.jbmt.2015.03.003.

Pedersen BK., Saltin B. 2015. Exercise as medicine - evidence for prescribing exercise as therapy in 26 different chronic diseases. Scandinavian Journal of Medicine \& Science in Sports 25:1-72. DOI: 10.1111/sms.12581.

Penelope L. 2002. Updating the principles of the Pilates method-Part 2. Journal of Bodywork and Movement Therapies 6:94-101. DOI: 10.1054/jbmt.2002.0289.

Pescatello LS., Arena A., Riebe D., Thompson PD. 2014. ACSM's guidelines for exercise testing and prescription. Philadelphia: Wolters Kluwer/Lippincott Williams \& Wilkins Health.

Reidpath DD., Cheah JC-H., Lam F-C., Yasin S., Soyiri I., Allotey P. 2013. Validity of selfmeasured waist and hip circumferences: results from a community study in Malaysia. Nutrition Journal 12:135. DOI: 10.1186/1475-2891-12-135.

Rogers K., Gibson AL. 2009. Eight-week traditional mat Pilates training-program effects on adult fitness characteristics. Research Quarterly for Exercise and Sport 80:569-574. DOI: 10.1080/02701367.2009.10599595.

Ross R., Dagnone D., Jones PJ., Smith H., Paddags A., Hudson R., Janssen I. 2000. Reduction in obesity and related comorbid conditions after diet-induced weight loss or exercise-induced weight loss in men. A randomized, controlled trial. Annals of Internal Medicine 133:92103.

Ross SE., Flynn JI., Pate RR. 2016. What is really causing the obesity epidemic? A review of reviews in children and adults. Journal of Sports Sciences 34:1148-1153. DOI: 10.1080/02640414.2015.1093650.

Ross R., Janssen I. 1999. Is abdominal fat preferentially reduced in response to exercise-induced 
766

767

768

769

770

771

772

773

774

775

776

777

778

779

780

781

782

783

784

785

786

787

788

789

790

791

792

793

794

795

796

weight loss? Medicine and Science in Sports and Exercise 31:S568-S572. DOI: 10.1097/00005768-199911001-00014.

Ruffino JS., Songsorn P., Haggett M., Edmonds D., Robinson AM., Thompson D., Vollaard NBJ. 2017. A comparison of the health benefits of reduced-exertion high-intensity interval training (REHIT) and moderate-intensity walking in type 2 diabetes patients. Applied Physiology, Nutrition, and Metabolism 42:202-208. DOI: 10.1139/apnm-2016-0497.

Santangelo KS., Radakovich LB., Fouts J., Foster MT. 2016. Pathophysiology of obesity on knee joint homeostasis: contributions of the infrapatellar fat pad. Hormone Molecular Biology and Clinical Investigation 26:97-108. DOI: 10.1515/hmbci-2015-0067.

Sarsan A., Ardiç F., Özgen M., Topuz O., Sermez Y. 2006. The effects of aerobic and resistance exercises in obese women. Clinical Rehabilitation 20:773-782. DOI: $10.1177 / 0269215506070795$.

Sarti MA., Monfort M., Fuster MA., Villaplana LA. 1996. Muscle activity in upper and lower rectus abdominus during abdominal exercises. Archives of Physical Medicine and Rehabilitation 77:1293-1297. DOI: 10.1016/S0003-9993(96)90195-1.

Şavkin R., Aslan UB. 2017. The effect of Pilates exercise on body composition in sedentary overweight and obese women. The Journal of Sports Medicine and Physical Fitness 57:1464-1470. DOI: 10.23736/S0022-4707.16.06465-3.

Segal NA., Hein J., Basford JR. 2004. The effects of Pilates training on flexibility and body composition: an observational study. Archives of Physical Medicine and Rehabilitation 85:1977-1981. DOI: 10.1016/j.apmr.2004.01.036.

Shinkai S., Watanabe S., Kurokawa Y., Torii J., Asai H., Shephard RJ. 1994. Effects of 12 weeks of aerobic exercise plus dietary restriction on body composition, resting energy expenditure and aerobic fitness in mildly obese middle-aged women. European Journal of Applied Physiology and Occupational Physiology 68:258-265. DOI: 10.1007/BF00376775.

Sparling PB., Millard-Stafford M., Snow TK. 1997. Development of a cadence curl-up test for college students. Research Quarterly for Exercise and Sport 68:309-316. DOI: 10.1080/02701367.1997.10608012.

Tremmel M., Gerdtham U-G., Nilsson P., Saha S. 2017. Economic burden of obesity: A systematic literature review. International Journal of Environmental Research and Public Health 14:435. DOI: 10.3390/ijerph14040435. 
797 Wells KF., Dillon EK. 1952. The sit and reach-A test of back and leg flexibility. Research 798 Quarterly for Exercise and Sport 23:115-118. DOI: 10.1080/10671188.1952.10761965.

799 Weltman A. 1995. The blood lactate responses to exercise. Illinois: Human Kinetics.

800 West SG., Duan N., Pequegnat W., Gaist P., Des Jarlais DC., Holtgrave D., Szapocznik J., 801 Fishbein M., Rapkin B., Clatts M., Mullen PD. 2008. Alternatives to the randomized 802 controlled trial. American journal of public health 98:1359-1366. DOI: $803 \quad$ 10.2105/AJPH.2007.124446.

804 Whipp BJ., Ward SA., Wasserman K. 1986. Respiratory markers of the anaerobic threshold. In: 805 Advances in Cardiology. 47-64. DOI: 10.1159/000413438.

806 Wing RR. 1999. Physical activity in the treatment of the adulthood overweight and obesity: 807 current evidence and research issues. Medicine and Science in Sports and Exercise 808 31:S547-S552. DOI: 10.1097/00005768-199911001-00010.

809 World Health Organization. 2012.Physical activity. Health Topics. Available at $810 \quad h t t p: / / w w w . w h o . i n t / t o p i c s / p h y s i c a l \_a c t i v i t y / e n /$.

811 World Health Organization. 2015.Obesity and overweight. Available at 812 http://www.who.int/mediacentre/factsheets/fs311/en/index.html.

813 Xiao T., Fu Y-F. 2015. Resistance training vs. aerobic training and role of other factors on the 814 exercise effects on visceral fat. European Review for Medical and Pharmacological 815 Sciences 19:1779-1784.

816

817 FIGURE LEGENDS

818 Figure 1. Flowchart showing the Consolidated Standards of Reporting Trials (CONSORT) of 819 participants through the study

820

821

TABLE LEGENDS

822 Table 1. List of Pilates exercises and apparatus

823 Table 2. Average heart rate and internal training load during training for Pilates and aerobic groups 824 Table 3. Effects of 8 weeks of Pilates or aerobic training on assessed variables

825 Table 4. Isokinetic strength measured at $60 \%$ s and at $240 \%$ s, pre- and post-training, for Pilates, 826 aerobic, and control groups 
827 Table 5. Effects of 8 weeks of Pilates or aerobic training on cardiorespiratory maximal treadmill 828 test results for Pilates, aerobic and control groups 


\section{Figure 1}

Flowchart showing the Consolidated Standards of Reporting Trials (CONSORT) of participants through the study.

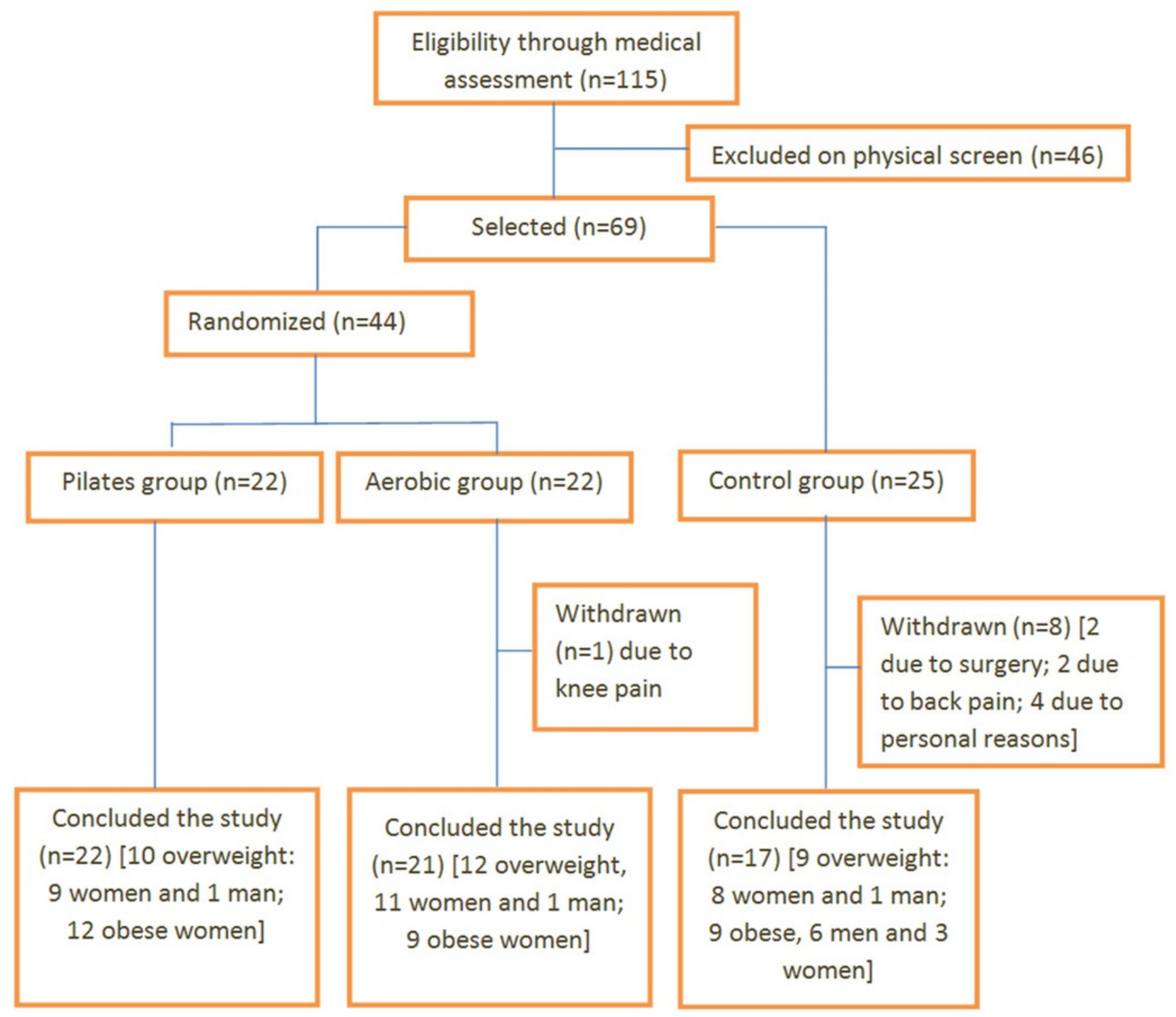




\section{Table $\mathbf{1}$ (on next page)}

List of Pilates exercises and apparatus

1-List of Pilates Exercises \& Equipment. Deborah Lessen. The PMA Pilates Certification Exam (Study Guide). 2-Brooke Siler, Christy Turlington. The Pilates Body: The Ultimate At-Home Guide to Strengthening, Lengthening and Toning Your Body - Without Machines. 2000. ISBN13:9780767903967. Details of the study protocol can be found on http://dx.doi.org/10.17504/protocols.io.p5wdq7e. 
1 Table 1. List of Pilates exercises and apparatus

\begin{tabular}{|c|c|c|}
\hline Name of exercises & $\begin{array}{l}\text { Number of } \\
\text { repetitions }\end{array}$ & $\begin{array}{l}\text { Evolution } \\
\text { in weeks }\end{array}$ \\
\hline \multicolumn{3}{|l|}{ Mat Pilates Program ${ }^{1}$} \\
\hline Hundred & 1 & 4 th-8th \\
\hline Single leg circles, single leg stretch, double leg stretch, spine & $3-5$ & 1 st -8 th \\
\hline \multicolumn{3}{|l|}{ Stretch, saw, side kick } \\
\hline Single leg kick, double leg kick, spine twist & $4-6$ & 2nd-8th \\
\hline Single straight leg stretch, criss-cross & $5-10$ & 4 th-8th \\
\hline Shoulder bridge, leg pull front & $3-6$ & 4 th-8th \\
\hline \multicolumn{3}{|l|}{ Program on Reformer ${ }^{1}$} \\
\hline Footwork, tendon stretch, pelvic lift, running, & $5-10$ & $1 \mathrm{st}-8 \mathrm{th}$ \\
\hline Short box series: round, flat back, twist & $3-5$ & 4 th -8 th \\
\hline Long box series: pulling straps, $\mathrm{T}$ & $5-8$ & 4 th -8 th \\
\hline Knee stretch series: round, arched back & $5-8$ & 4th-8th \\
\hline Long stretch series: front, elephant, down stretch & $5-8$ & 4 th-8th \\
\hline Chest expansion kneeling, reverse chest expansion, splits side, & $5-8$ & 4 th-8th \\
\hline
\end{tabular}

stomach massage, flat back, reach, twist

\section{Program on Cadillac ${ }^{1}$}

Roll down bar, breathing, chest expansion, thigh stretch

3-5 1st-8th

Push through seated front, spread eagle

3-5 1st-8th

Arm spring series (supine), leg spring series (supine)

3-5 1st-8th

Side lying series

5-10 4th-8th

Standing on floor: punching, salute, hug-a-tree, butterfly

3-6 4th-8th

\section{Program on Chair ${ }^{1}$}

Double leg pumps, single leg pumps, standing leg pump front,

5-10 1st-8th side, crossover

Washer woman, swan front, seated mermaid

3-5 4th-8th

Frog lying flat, single leg pump lying flat, Achilles stretch

5-10 1st-8th

\section{Program on Ladder Barrel $^{2}$}

Horse back 
Short box series: round, flat back and twist

3-5

4th-8th

\section{Program with Dumbbells-1 kg $^{2}$}

Wall: circles, sliding, rolling down

3-5 1st-8th

Zip-up, chest expansion, shaving the head, arm circles, biceps

5-10 2nd-8th

curls (I, II), triceps extension, the bug, boxing, lunges

\section{Program with Magic Circle ${ }^{1}$}

Arm work, leg work and head work series

5-10 1st-8th

2 1-List of Pilates Exercises \& Equipment. Deborah Lessen. The PMA Pilates Certification Exam

3 (Study Guide). 2-Brooke Siler, Christy Turlington. The Pilates Body: The Ultimate At-Home

4 Guide to Strengthening, Lengthening and Toning Your Body - Without Machines. 2000. ISBN-

5 13:9780767903967. Details of the study protocol can be found on

6 http://dx.doi.org/10.17504/protocols.io.p5wdq7e. 


\section{Table 2 (on next page)}

Average heart rate and internal training load during training for Pilates and aerobic groups

Data are mean \pm standard deviation. HR, heart rate; bpm, beats per minute; $d$ : Cohen $d$ effect size; $\mathrm{Cl}$ : confidence interval. *Aerobic $>$ Pilates. 
1 Table 2. Average heart rate and internal training load during training for Pilates and aerobic groups

\begin{tabular}{ccccccc}
\hline Variables & $\begin{array}{c}\text { Pilates } \\
(\mathrm{n}=22)\end{array}$ & $\begin{array}{c}\text { Aerobic } \\
(\mathrm{n}=21)\end{array}$ & $\Delta \%$ & $\mathrm{p}$ value & $d$ value & $90 \%$ CI \\
\hline Average HR $(\mathrm{bpm})$ & $86 \pm 6$ & $123 \pm 11^{*}$ & +42.2 & $<0.0001$ & 4.1 & $2.5-5.7$ \\
Internal training load & $192 \pm 69$ & $252 \pm 106^{*}$ & +31.3 & 0.04 & 0.67 & $0.14-1.2$
\end{tabular}

2 Data are mean \pm standard deviation. HR, heart rate; bpm, beats per minute; $d$ : Cohen $d$ effect size;

3 CI: confidence interval.

4 *Aerobic $>$ Pilates. 


\section{Table 3 (on next page)}

Effects of 8 weeks ofPilatesor aerobic training on variables assessed.

Data are mean \pm standard deviation. $B M I$, body mass index; $d$, Cohen $d$ effect size; $\mathrm{Cl}$, confidence interval. ${ }^{*} p<0.05$ (different from the pre-training for the same group). ${ }^{*} p<0.05$ (pre-training values for Controlgroup $\neq$ pre-training values forPilatesgroup). ${ }^{\star} p<0.05$ (pretraining values for Aerobicgroup $\neq$ pre-training values forPilatesand Control groups). ${ }^{\circledR} p<0.05$ (pos-training values for Controlgroup $\neq$ pos-training values forPilatesandAerobicgroups). + $p<0.05$ (pos-training values for Controlgroup $\neq$ pos-training values forPilatesgroup). 
Table 3. Effects of 8 weeks of Pilates or aerobic training on variables assessed.

\begin{tabular}{|c|c|c|c|c|c|c|}
\hline & Pre & Post & $\Delta \%$ & $\mathrm{p}$ value & $d$ value & $90 \% \mathrm{CI}$ \\
\hline \multicolumn{7}{|l|}{ Pilates group $(\mathrm{n}=22)$} \\
\hline BMI $\left(\mathrm{kg} / \mathrm{m}^{2}\right)$ & $31.9 \pm 3.9$ & $31.6 \pm 4.0^{*}$ & $-1.1 \pm 1.8$ & 0.009 & 0.07 & $0.01-0.13$ \\
\hline Total body mass (kg) & $81.0 \pm 10.0$ & $80.8 \pm 10.2$ & $-0.5 \pm 1.4$ & 0.19 & 0.01 & $-0.01-0.03$ \\
\hline Fat mass $(\%)$ & $50.9 \pm 6.1^{\#}$ & $49.1 \pm 5.8^{*}$ & $-3.7 \pm 5.2$ & 0.0001 & 0.19 & $0.11-0.27$ \\
\hline Lean mass $(\mathrm{kg})$ & $38.4 \pm 6.5$ & $39.7 \pm 6.6^{*}$ & $3.2 \pm 5.0$ & 0.0005 & 0.19 & $0.11-0.27$ \\
\hline Waist circumference $(\mathrm{cm})$ & $105.6 \pm 9.8$ & $102.9 \pm 9.9 *$ & $-2.6 \pm 2.9$ & 0.0009 & 0.27 & $0.14-0.40$ \\
\hline Hip circumference $(\mathrm{cm})$ & $115.7 \pm 9.4$ & $114.0 \pm 10.1^{*}$ & $-1.5 \pm 1.6$ & 0.0001 & 0.17 & $0.1-0.24$ \\
\hline Abdominal test $\left(\mathrm{n}^{\mathrm{o}}\right.$ of & $30.7 \pm 6.3$ & $41.6 \pm 6.6^{*}$ & $25.6 \pm 13.5$ & 0.0001 & 1.69 & $1.0-2.40$ \\
\hline \multicolumn{7}{|l|}{ repetitions) } \\
\hline Trunk extensor test (s) & $89.5 \pm 42.8$ & $129.4 \pm 55.7 *$ & $28.7 \pm 23.6$ & 0.0003 & 0.80 & $0.46-1.10$ \\
\hline Chair test (s) & $24.5 \pm 3.3$ & $19.6 \pm 3.3^{*}$ & $-26.9 \pm 27.9$ & 0.0001 & 1.48 & $0.89-2.10$ \\
\hline Stair test (s) & $25.3 \pm 3.4$ & $22.8 \pm 3.0^{*}$ & $-9.8 \pm 7.9$ & 0.003 & 0.78 & $0.40-1.20$ \\
\hline Flexibility $(\mathrm{cm})$ & $21.5 \pm 10.7$ & $25.6 \pm 9.4^{*}$ & $21.8 \pm 21.6$ & 0.0001 & 0.41 & $0.25-0.57$ \\
\hline \multicolumn{7}{|l|}{ Aerobic group $(n=21)$} \\
\hline BMI $\left(\mathrm{kg} / \mathrm{m}^{2}\right)$ & $30.3 \pm 3.3$ & $30.1 \pm 3.4$ & $-0.6 \pm 1.8$ & 0.12 & 0.05 & $-0.01-0.10$ \\
\hline Total body mass (kg) & $77.7 \pm 10.6$ & $77.4 \pm 10.6$ & $-0.5 \pm 1.8$ & 0.20 & 0.03 & $-0.01-0.07$ \\
\hline Fat mass $(\%)$ & $46.4 \pm 6.2$ & $45.9 \pm 6.4$ & $-1.2 \pm 2.4$ & 0.60 & 0.05 & $-0.03-0.14$ \\
\hline Lean mass $(\mathrm{kg})$ & $40.0 \pm 5.5$ & $40.2 \pm 5.6$ & $0.5 \pm 2.7$ & 0.49 & 0.03 & $-0.04-0.10$ \\
\hline Waist circumference $(\mathrm{cm})$ & $102.7 \pm 8.4$ & $99.5 \pm 8.8^{*}$ & $-3.3 \pm 3.7$ & 0.0001 & 0.37 & $0.22-0.52$ \\
\hline Hip circumference $(\mathrm{cm})$ & $111.6 \pm 7.2$ & $110.6 \pm 7.3^{*}$ & $-0.8 \pm 1.0$ & 0.001 & 0.14 & $0.07-0.21$ \\
\hline
\end{tabular}




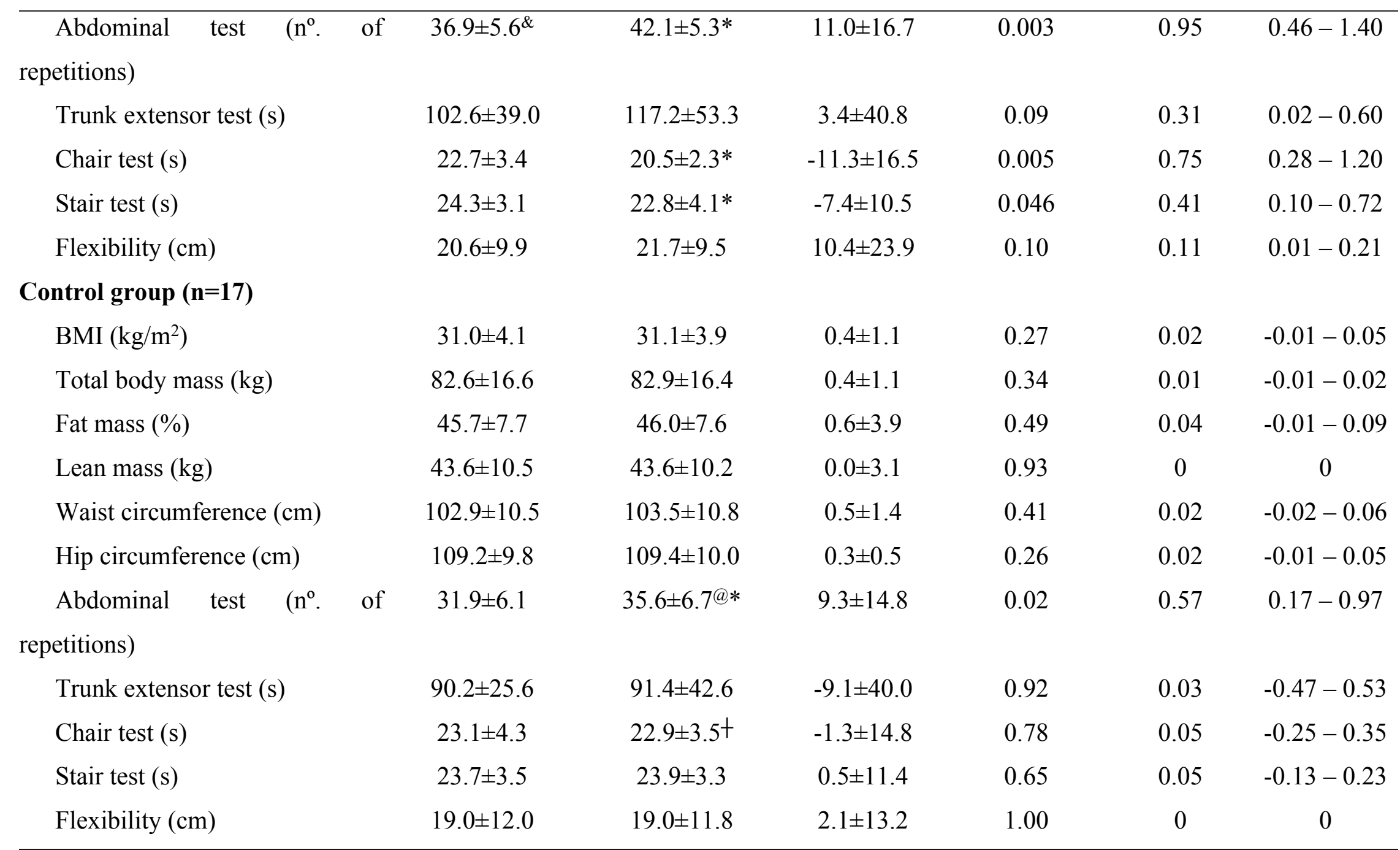

2 Data are mean \pm standard deviation. BMI, body mass index; $d$, Cohen $d$ effect size; CI, confidence interval.

$3 * \mathrm{p}<0.05$ (different from the pre-training for the same group).

$4 \quad{ }^{\#} \mathrm{p}<0.05$ (pre-training values for Control group $\neq$ pre-training values for Pilates group). 
$5 \quad \&_{\mathrm{p}}<0.05$ (pre-training values for Aerobic group $\neq$ pre-training values for Pilates and Control groups).

$6 a_{\mathrm{p}}<0.05$ (pos-training values for Control group $\neq$ pos-training values for Pilates and Aerobic groups).

$7+\mathrm{p}<0.05$ (pos-training values for Control group $\neq$ pos-training values for Pilates group). 


\section{Table 4 (on next page)}

Isokinetic strength measured at $60 \% \mathrm{~s}$ and at $240 \% \mathrm{~s}$, pre- and post-training, for Pilates, aerobic, and control groups

Data are mean \pm standard deviation. PT, peak torque; BM, body mass; $d$, Cohen $d$ effect size;

$\mathrm{Cl}$, confidence interval. ${ }^{*} p<0.05$ (pre vs. post). $\# p<0.05$ (Pilates vs. Control). 
1 Table 4. Isokinetic strength measured at $60 \%$ s and at $240 \%$, pre- and post-training, for Pilates, aerobic, and control groups.

\begin{tabular}{|c|c|c|c|c|c|c|}
\hline Variables & Pre & Post & $\Delta \%$ & $\mathrm{p}$ value & $d$ values & $90 \% \mathrm{CI}$ \\
\hline \multicolumn{7}{|l|}{ Pilates $(\mathrm{n}=22)$} \\
\hline \multicolumn{7}{|l|}{$60 \% / \mathrm{s}$} \\
\hline PT/BM extensor dominant (\%) & $128.9 \pm 37.6^{\#}$ & $138.6 \pm 36.2$ & $5.5 \pm 12.2$ & 0.34 & 0.26 & $-0.19-0.71$ \\
\hline PT/BM extensor non-dominant (\%) & $124.4 \pm 36.2$ & $119.2 \pm 39.3$ & $-0.9 \pm 15.5$ & 0.83 & 0.13 & $-0.88-1.10$ \\
\hline PT/BM flexor dominant (\%) & $65.7 \pm 27.4$ & $72.6 \pm 24.7$ & $5.0 \pm 28.6$ & 1.00 & 0.26 & $-34.0-35.0$ \\
\hline PT/BM flexor non-dominant (\%) & $62.6 \pm 19.3$ & $67.4 \pm 16.5$ & $5.1 \pm 17.9$ & 0.98 & 0.25 & $-16.0-17.0$ \\
\hline \multicolumn{7}{|l|}{$240^{\circ} / \mathrm{s}$} \\
\hline PT/BM extensor dominant $(\%)$ & $75.8 \pm 21.9^{\#}$ & $76.2 \pm 23.7$ & $6.6 \pm 13.1$ & 0.83 & 0.02 & $-0.14-0.18$ \\
\hline PT/BM extensor non-dominant (\%) & $72.4 \pm 21.5^{\#}$ & $74.3 \pm 18.1$ & $2.1 \pm 19.3$ & 0.58 & 0.01 & $-0.02-0.04$ \\
\hline PT/BM flexor dominant (\%) & $65.8 \pm 27.4$ & $51.1 \pm 14.0$ & $8.3 \pm 22.2$ & 0.50 & 0.67 & $-0.98-2.30$ \\
\hline PT/BM flexor non-dominant (\%) & $62.6 \pm 19.3$ & $46.3 \pm 12.4$ & $7.9 \pm 19.1$ & 0.29 & 1.00 & $-0.57-2.60$ \\
\hline \multicolumn{7}{|l|}{ Aerobic $(n=21)$} \\
\hline \multicolumn{7}{|l|}{$60 \% / \mathrm{s}$} \\
\hline PT/BM extensor dominant (\%) & $146.5 \pm 25.0$ & $146.4 \pm 24.2$ & $-1.1 \pm 15.9$ & 1.00 & 0.00 & 0 \\
\hline PT/BM extensor non-dominant (\%) & $137.5 \pm 38.1$ & $135.9 \pm 36.2$ & $-2.2 \pm 16.5$ & 0.99 & 0.04 & $-5.30-5.40$ \\
\hline PT/BM flexor dominant (\%) & $75.5 \pm 28.9$ & $72.6 \pm 19.1$ & $-5.7 \pm 27.4$ & 0.99 & 0.12 & $-16.0-16.0$ \\
\hline PT/BM flexor non-dominant (\%) & $63.1 \pm 19.1$ & $68.9 \pm 17.8$ & $8.2 \pm 16.1$ & 0.99 & 0.31 & $-41.0-41.0$ \\
\hline \multicolumn{7}{|l|}{$240^{\circ} / \mathrm{s}$} \\
\hline PT/BM extensor dominant (\%) & $90.3 \pm 14.7$ & $89.2 \pm 16.9$ & $-2.2 \pm 10.9$ & 0.98 & 0.07 & $-4.60-4.70$ \\
\hline PT/BM extensor non-dominant (\%) & $87.0 \pm 18.5$ & $85.3 \pm 20.6$ & $-4.5 \pm 20.9$ & 0.17 & 0.08 & $-0.02-0.18$ \\
\hline
\end{tabular}




\begin{tabular}{lcccccc}
\hline PT/BM flexor dominant (\%) & $52.8 \pm 9.5$ & $55.7 \pm 14.4$ & $2.4 \pm 19.2$ & 0.84 & 0.23 & $-1.70-2.10$ \\
PT/BM flexor non-dominant (\%) & $52.5 \pm 10.0$ & $50.9 \pm 10.9$ & $-5.5 \pm 19.3$ & 0.95 & 0.15 & $-3.80-4.10$
\end{tabular}

\section{Control $(\mathbf{n}=\mathbf{1 7})$}

$60 \% \mathrm{~s}$

$\begin{array}{lcccccc}\text { PT/BM extensor dominant (\%) } & 165.9 \pm 36.6 & 163.6 \pm 38.3 & -3.0 \pm 112 & 0.99 & 0.06 & -7.90-8.00 \\ \text { PT/BM extensor non-dominant (\%) } & 157.1 \pm 34.8 & 161.2 \pm 38.9 & 0.3 \pm 13.7 & 0.98 & 0.11 & -7.20-7.40 \\ \text { PT/BM flexor dominant (\%) } & 76.9 \pm 18.5 & 80.4 \pm 20.8 & 2.4 \pm 11.4 & 0.89 & 0.18 & -2.00-2.30 \\ \text { PT/BM flexor non-dominant (\%) } & 74.2 \pm 21.6 & 74.4 \pm 22.5 & -0.9 \pm 16.3 & 0.32 & 0.01 & -0.01-0.02\end{array}$

$240 \% \mathrm{~s}$

$\begin{array}{lllllll}\text { PT/BM extensor dominant (\%) } & 99.0 \pm 19.9 & 103.5 \pm 21.4 & 3.7 \pm 6.9 & 0.98 & 0.22 & -14.0-15.0 \\ \text { PT/BM extensor non-dominant (\%) } & 95.6 \pm 18.9 & 102.4 \pm 22.9 & 4.9 \pm 10.4 & 1.00 & 0.32 & -42.0-43.0 \\ \text { PT/BM flexor dominant (\%) } & 53.7 \pm 15.5 & 55.5 \pm 11.4 & 2.9 \pm 12.2 & 0.98 & 0.13 & -8.50-8.80 \\ \text { PT/BM flexor non-dominant (\%) } & 53.6 \pm 12.6 & 53.3 \pm 13.4 & -3.0 \pm 20.6 & 0.99 & 0.02 & -2.60-2.70\end{array}$

2 Data are mean \pm standard deviation. PT, peak torque; BM, body mass; $d$, Cohen $d$ effect size; CI, confidence interval.

$3 * \mathrm{p}<0.05$ (pre vs. post).

$4 \quad \# \mathrm{p}<0.05$ (Pilates vs. Control). 


\section{Table 5 (on next page)}

Effects of 8 weeks of Pilates or aerobic training on cardiorespiratory maximal treadmill test results for Pilates, Aerobic and Control groups.

Data are mean \pm standard deviation. $\mathrm{V}<$ !--[if !msEquation]-- $<<$ !--[endif]-- $>\mathrm{O}_{2}$, oxygen uptake; $\mathrm{VO}_{2} \max$, maximal oxygen uptake; $\mathrm{HR}$, heart rate; HRmax, maximal heart rate; VT, ventilatory threshold; RCP, respiratory compensation point; $d$, Cohen $d$ effect size; $\mathrm{Cl}$, confidence interval. ${ }^{*} p<0.05$ (pre vs. post). ${ }^{*} p<0.05$ (Pilates $<$ Aerobic and Control). 
Table 5. Effects of 8 weeks of Pilates or aerobic training on cardiorespiratory maximal treadmill test results for Pilates, Aerobic and

2 Control groups.

\begin{tabular}{|c|c|c|c|c|c|c|}
\hline Variables & Pre & Post & $\Delta \%$ & $p$ value & $d$ value & $90 \% \mathrm{CI}$ \\
\hline \multicolumn{7}{|l|}{ Pilates $(\mathrm{n}=22)$} \\
\hline$\dot{V} \mathrm{O}_{2}$ in $\mathrm{VT}(\mathrm{mL} / \mathrm{kg} / \mathrm{min})$ & $15.0 \pm 2.6$ & $17.2 \pm 3.4^{*}$ & $11.6 \pm 17.6$ & $<0.01$ & 0.60 & $0.31-0.89$ \\
\hline HR in VT (bpm) & $109 \pm 13^{\#}$ & $122 \pm 15^{*}$ & $10 \pm 11$ & $<0.01$ & 0.90 & $0.47-1.30$ \\
\hline$\dot{V} \mathrm{O}_{2}$ in $\mathrm{RCP}(\mathrm{mL} / \mathrm{kg} / \mathrm{min})$ & $19.4 \pm 3.6$ & $21.1 \pm 4.1^{*}$ & $7.9 \pm 19.5$ & 0.01 & 0.48 & $0.18-0.78$ \\
\hline HR in RCP (bpm) & $130 \pm 15^{\#}$ & $139 \pm 15$ & $6.0 \pm 9.6$ & 0.01 & 0.56 & $0.21-0.91$ \\
\hline$\dot{V} \mathrm{O}_{2} \max (\mathrm{mL} / \mathrm{kg} / \mathrm{min})$ & $22.4 \pm 4.9$ & $24.2 \pm 4.5^{*}$ & $19.9 \pm 15.5$ & 0.01 & 0.33 & $0.12-0.54$ \\
\hline HRmax (bpm) & $146 \pm 21^{\#}$ & $155 \pm 16^{*}$ & $6.2 \pm 11.7$ & $<0.01$ & 0.48 & $0.24-0.72$ \\
\hline \multicolumn{7}{|l|}{ Aerobic $(n=21)$} \\
\hline$\dot{V} \mathrm{O}_{2}$ in $\mathrm{VT}(\mathrm{mL} / \mathrm{kg} / \mathrm{min})$ & $18.7 \pm 2.7$ & $20.1 \pm 3.2$ & $6.1 \pm 10.2$ & 0.22 & 0.38 & $0.05-0.71$ \\
\hline HR in VT (bpm) & $126 \pm 10$ & $127 \pm 17$ & $-0.7 \pm 16.6$ & 0.93 & 0.09 & $-0.16-1.8$ \\
\hline$\dot{V} \mathrm{O}_{2}$ in $\mathrm{RCP}(\mathrm{mL} / \mathrm{kg} / \mathrm{min})$ & $23.2 \pm 3.8$ & $24.3 \pm 3.8$ & $3.0 \pm 12.3$ & 0.72 & 0.21 & $-0.13-0.55$ \\
\hline $\mathrm{HR}$ in RCP (bpm) & $146 \pm 16$ & $145 \pm 19$ & $-1.8 \pm 11.0$ & 0.91 & 0.03 & $-0.09-0.15$ \\
\hline$\dot{V} \mathrm{O}_{2} \max (\mathrm{mL} / \mathrm{kg} / \mathrm{min})$ & $26.8 \pm 4.3$ & $27.0 \pm 4.4$ & $14.8 \pm 10.5$ & 0.64 & 0.13 & $-0.11-0.37$ \\
\hline HRmax (bpm) & $160 \pm 16$ & $158 \pm 19$ & $-1.7 \pm 6.6$ & 0.48 & 0.12 & $-0.16-0.40$ \\
\hline \multicolumn{7}{|l|}{ Control $(n=17)$} \\
\hline$\dot{V} \mathrm{O}_{2}$ in $\mathrm{VT}(\mathrm{mL} / \mathrm{kg} / \mathrm{min})$ & $17.9 \pm 3.9$ & $18.7 \pm 4.3$ & $3.4 \pm 14.7$ & 0.18 & 0.19 & $-0.04-0.42$ \\
\hline HR in VT (bpm) & $127 \pm 11$ & $128 \pm 10$ & $0.4 \pm 10.0$ & 0.94 & 0.10 & $-2.10-2.30$ \\
\hline$\dot{V} \mathrm{O}_{2}$ in $\mathrm{RCP}(\mathrm{mL} / \mathrm{kg} / \mathrm{min})$ & $22.3 \pm 4.9$ & $22.4 \pm 4.5$ & $-1.2 \pm 10.1$ & 0.73 & 0.04 & $-0.15-0.23$ \\
\hline HR in RCP (bpm) & $146 \pm 13$ & $145 \pm 10$ & $-0.8 \pm 6.3$ & 0.92 & 0.05 & $-0.78-0.88$ \\
\hline
\end{tabular}




$\begin{array}{lcccccc}\dot{\mathrm{V}} \mathrm{O}_{2} \max (\mathrm{mL} / \mathrm{kg} / \mathrm{min}) & 25.9 \pm 5.4 & 25.2 \pm 4.7 & 9.8 \pm 11.5 & 0.31 & 0.08 & -0.05-0.21 \\ \mathrm{HRmax}(\mathrm{bpm}) & 165 \pm 12 & 156 \pm 12^{*} & -6.2 \pm 6.5 & 0.02 & 0.74 & 0.22-1,30\end{array}$

$3 \quad$ Data are mean \pm standard deviation. $\dot{V} \mathrm{O}_{2}$, oxygen uptake; $\dot{V} \mathrm{O}_{2}$ max, maximal oxygen uptake; HR, heart rate; HRmax, maximal heart

4 rate; VT, ventilatory threshold; RCP, respiratory compensation point; $d$, Cohen $d$ effect size; CI, confidence interval.

$5 \quad * \mathrm{p}<0.05$ (pre vs. post).

$6{ }^{\#} \mathrm{p}<0.05$ (Pilates $<$ Aerobic and Control). 